$12-1-2019$

\title{
Les pharmacies privées dans l'offre de services de planification familiale au Sénégal : Une étude pilote
}

\author{
Fatou Mbow \\ Population Council \\ Dela Nai \\ Population Council \\ Sara Chace Dwyer \\ Population Council \\ Aparna Jain \\ Population Council \\ Babacar Mane \\ Population Council
}

Follow this and additional works at: https://knowledgecommons.popcouncil.org/departments_sbsr-rh

Part of the International Public Health Commons, and the Social and Behavioral Sciences Commons How does access to this work benefit you? Let us know!

\section{Recommended Citation}

Mbow, Fatou, Dela Nai, Sara Chace Dwyer, Aparna Jain, and Babacar Mane. 2019. "Les pharmacies privées dans l'offre de services de planification familiale au Sénégal : Une étude pilote." Dakar: Population Council, The Evidence Project. 
Les pharmacies privées dans l'offre de services de planification familiale au Sénégal : Une étude pilote

Fatou Bintou Mbow, Population Council

Dela Nai, Population Council

Sara Chace Dwyer, Population Council

Aparna Jain, Population Council

Babacar Mané, Consultant Indépendant Sénégal

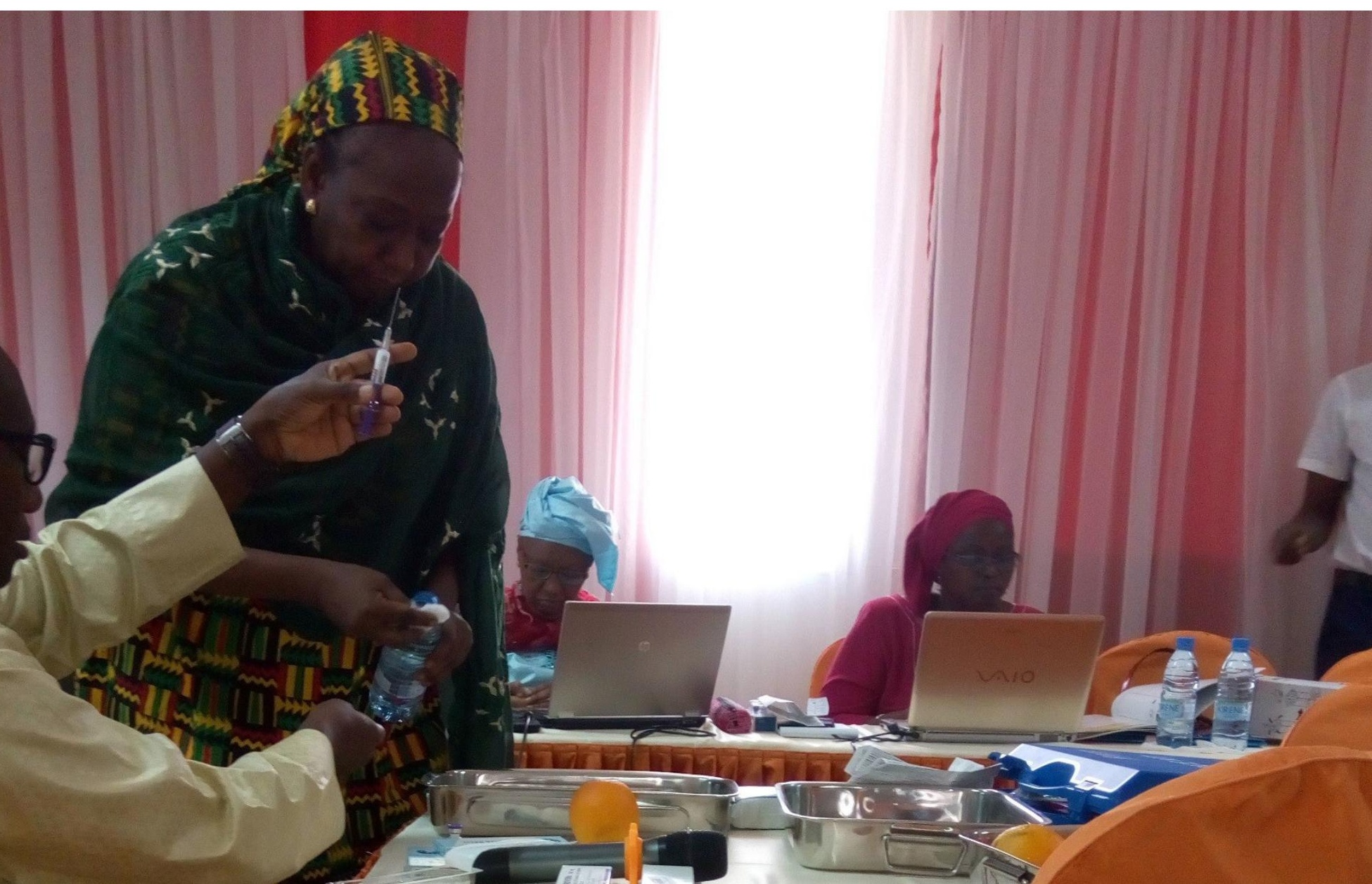




\section{Le Projet Evidence \\ Population Council}

4301 Connecticut Avenue, NW, Suite 280

Washington, DC 20008 USA

tel +12022379400

evidenceproject.popcouncil.org

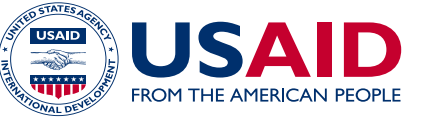

Le Projet Evidence est rendu possible grâce au généreux support du Peuple Américain à travers l'Agence des Etats Unis pour le Développement International (USAID) sous les termes de l'accord de coopération no. AID-OAA-A-13-00087. Le contenu de ce document est la seule responsabilité du Projet Evidence et ne reflète pas nécessairement les points de vue de l'USAID ou du Gouvernement des Etats Unis.

Evidence

Le Projet Evidence utilise la science de la mise en œuvre - la production, traduction, et utilisation stratégique d'évidences — pour renforcer et mettre à l'échelle des programmes de planification familiale et de santé de la reproduction afin de réduire les grossesses non désirées partout dans le monde. Le Projet Evidence est dirigé par le Population Council.

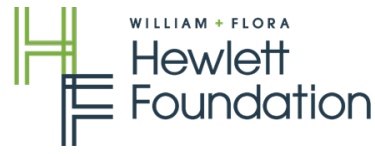

La Fondation William et Flora Hewlett est une fondation de bienfaisance non partisane et privée qui avance des idées et soutient les institutions pour promouvoir un monde meilleur.

Publié en Décembre 2019

Citation recommandée : Mbow, Fatou Bintou, Dela Nai, Sara Chace Dwyer, Aparna Jain, Babacar Mané. 2019. «Les pharmacies privées dans l'offre de services de planification familiale au Sénégal : Une étude pilote. » Dakar, Sénégal : Population Council, Le Projet Evidence.

Crédit photo sur la page de garde : Population Council

C2019 Le Population Council, Inc. 


\section{Table des Matières}

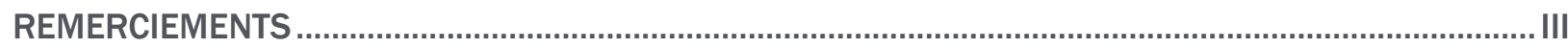

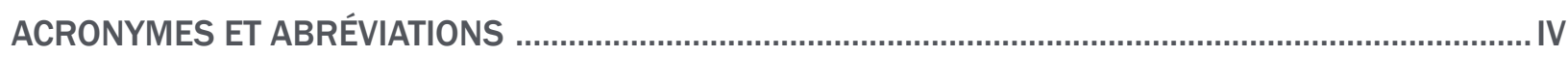

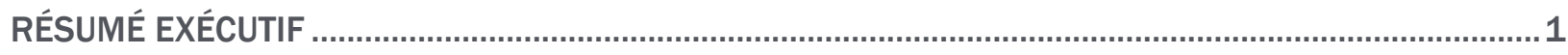

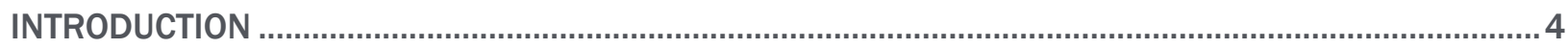

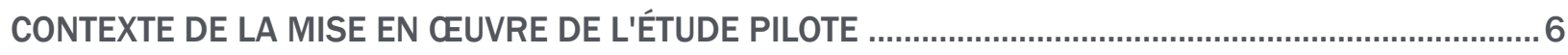

Révisions du protocole..............................................................................................................

Révision des outils et de la durée de la formation ............................................................................

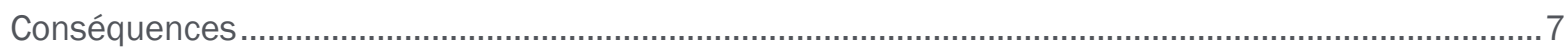

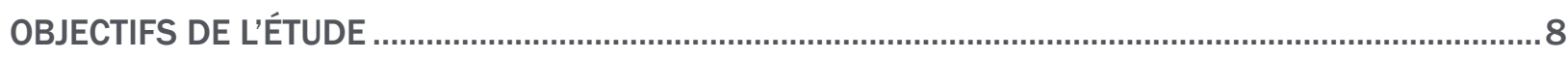

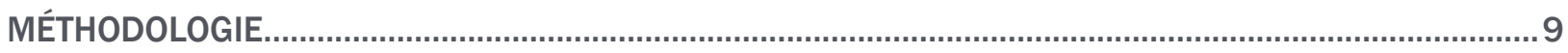

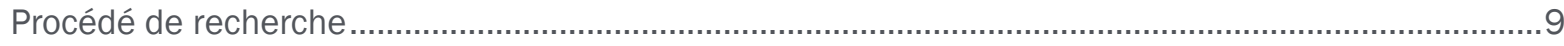

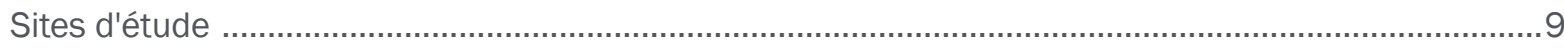

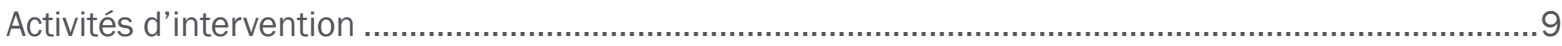

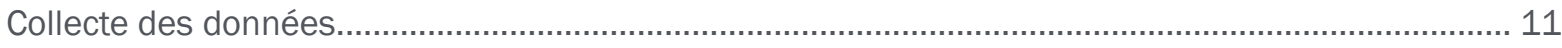

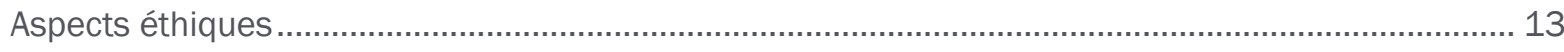

Gestion et analyse des données................................................................................................. 13

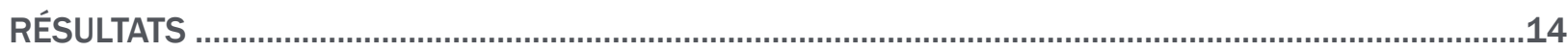

Profil du personnel de pharmacie ................................................................................................ 14

Résultat 1: Connaissances et compétences du personnel de pharmacies.......................................... 15

Résultat 2 : Qualité du counseling en PF à travers des visites de clientes mystères ........................... 23

Résultat 3 : Offre des services en PF et gestion des données .............................................................24

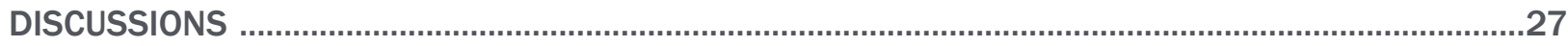

Potentiel de counseling en PF dans les pharmacies privées avec un soutien supplémentaire

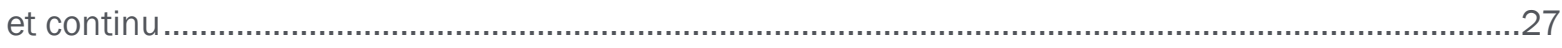

Les pharmaciens et les pharmaciens assistants ont besoin d'un soutien supplémentaire pour mieux offrir des contraceptifs oraux et injectables.......................................................................27

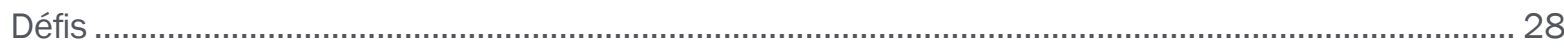

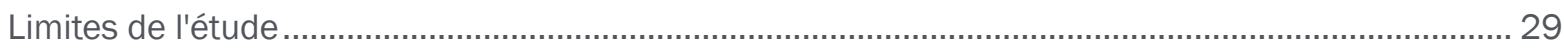

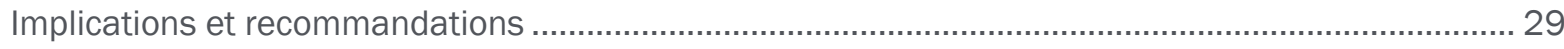

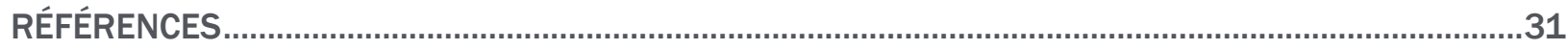

ANNEXE 1

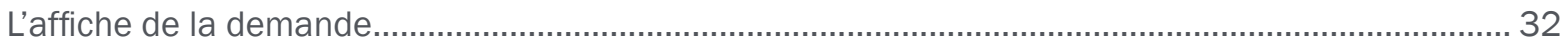




\section{LISTE DES FIGURES}

Figure 1 : Connaissances du personnel des pharmacies privées sur les méthodes contraceptives, au pré-test et au post-test, $\mathrm{N}=69$

Figure 2 : Connaissances générales pour le counseling en PF du personnel des pharmacies, au pré-test et au post-test, $\mathrm{N}=69$.

Figure 3 : Perception de la durée du counseling en PF parmi le personnel qui a fait le counseling en $\mathrm{PF}$ après la formation, $\mathrm{n}=37$.

\section{LISTE DES TABLEAUX}

Tableau 1 : Caractéristiques du personnel de pharmacies privées au pré-test, N=69

Tableau 2 : Expérience d'offre des services de a PF au pré-test, $\mathrm{N}=69$.

Tableau 3 : Connaissances du personnel des pharmacies privées sur les questions clés pendant une séance de counseling, au pré-test et au post-test, $\mathrm{N}=69$

Tableau 4 : Connaissances du personnel des pharmacies privées sur les questions pour écarter le doute d'une grossesse, au pré-test et au post-test, $\mathrm{N}=69$

Tableau 5 : Connaissance des pharmaciens et des pharmaciens assistants sur les effets secondaires courants du contraceptif oral combiné, au pré-test et au post-test, $n=23$.

Tableau 6 : Connaissance des pharmaciens et des pharmaciens assistants sur les effets secondaires courants des contraceptifs oraux à progestatif seul, au pré-test et au post-test, $n=23$

Tableau 7 : Connaissance des pharmaciens et des pharmaciens assistants sur les signes avant-coureurs d'un contraceptif oral qui exigent qu'une cliente se fasse soigner dans un établissement de santé, au pré-test et au post-test, $n=23$.

Tableau 8 : Connaissance des pharmaciens et des pharmaciens assistants sur les conditions qui limitent l'utilisation des contraceptifs oraux, au pré-test et au post-test, $n=23$.

Tableau 9 : Connaissance de pharmaciens et de pharmaciens assistants sur les instructions à donner à une femme si elle oublie de prendre une pilule " active " 1 ou 2 jours d'affilée, au pré-test et au post-test, n=23 ....

Tableau 10 : Connaissance des pharmaciens et des pharmaciens assistants sur les instructions à une femme si elle oublie une pilule " active " 3 jours ou plus d'affilée, au pré-test et au post-test, $n=23$.

Tableau 11 : Connaissance des pharmaciens et des pharmaciens assistants sur les effets secondaires courants des contraceptifs injectables, au pré-test et au post-test, $n=23$.

Tableau 12 : Connaissance des pharmaciens et des pharmaciens assistants sur les conditions qui limitent l'utilisation des contraceptifs injectables, au pré-test et au post-test, $n=23$.

Tableau 13 : Connaissance des pharmaciens et des pharmaciens assistants sur le moment où une femme peut commencer à utiliser des méthodes injectables à progestatif-seul, au pré-test et au post-test, n=23

Tableau 14 : Connaissance des pharmaciens et des pharmaciens assistants sur le mode d'administration et du dispositif d'injection du DMPA-IM et DMPA-SC, au pré-test et au post-test, $\mathrm{n}=\mathbf{2 3}$.

Tableau 15 : Connaissance des pharmaciens et des pharmaciens assistants sur les endroits sur le corps où le DMPA-IM et DMPA-SC sont administrés, au pré-test et au post-test, $\mathrm{n}=\mathbf{2 3}$

Tableau 16 : Profil du personnel des pharmacies rencontré lors des visites de clientes mystères, $\mathrm{N}=51$

Tableau 17 : L'endroit du counseling de la PF, selon les visites des clientes mystères, N=51

Tableau 18 : Les aspects visant la qualité des services dans le processus du counseling reçu par les clientes mystères lors de leur visites, $\mathrm{N}=51$

Tableau 19 : Aspects divers de counseling en PF reçus par les clientes mystères, $\mathrm{N}=51$

Tableau 20 : Offre des services de la PF, enquête du suivi, $n=68$ 


\section{Remerciements}

Le Population Council adresse ses vifs remerciements à tous ceux qui ont contribué à la réalisation de cette étude, notamment, le Ministère de la Santé et de l'Action Sociale, à travers la Direction de la Santé de la Mère et de l'Enfant (DSME), partenaire clé de cette recherche qui a contribué à la conception, à la mise en œuvre et au suivi de cette étude.

Nos remerciements vont aussi à l'endroit de l'Ordre et du syndicat des pharmaciens qui ont appuyé le processus et facilité la mise en œuvre de la collecte des données sur le terrain.

Nous exprimons notre profonde gratitude à l'endroit des propriétaires et personnels des pharmacies que nous avons visitées, pour leur disponibilité lors de la préparation et de la mise en ouvre de l'étude et à toutes les personnes qui ont bien voulu prendre de leur temps et se prêter à nos interviews.

Nous adressons aussi nos vifs remerciements aux partenaires techniques qui ont contribué à une bonne mise en œuvre de l'intervention, notamment Marie Stopes International et PATH pour la mise à disposition respective de « choice kits » et de placebo de Sayana ${ }^{\circledR}$ Press lors des sessions de formation.

Nous saluons ici le professionnalisme des formateurs (trices), agents de collecte et agents de saisie qui n'ont ménagé aucun effort pour la réussite de l'étude.

Cette étude a pu se réaliser grâce au financement de l'USAID à travers Le Projet Evidence, et l'appui de la Fondation William et Flora Hewlett à travers un financement au Population Council. 


\section{Acronymes et abréviations}

$\begin{array}{ll}\text { BCS+ } & \text { Balanced Counseling Strategy Plus } \\ \text { CSNPF } & \text { Cadre stratégique national pour la planification familiale } \\ \text { DMPA-IM } & \text { Acétate dépôt médroxyprogestérone- intramusculaire } \\ \text { DMPA-SC } & \text { Acétate dépôt médroxyprogestérone-sous-cutanée } \\ \text { DSME } & \text { Direction de la santé de la mère et de l'enfant } \\ \text { GdS } & \text { Gouvernement du Sénégal } \\ \text { IST } & \text { Infections sexuellement transmissibles } \\ \text { MSAS } & \text { Ministère de la Santé et de l'action Sociale } \\ \text { OMS } & \text { Organisation mondiale de la santé } \\ \text { OPS } & \text { Ordre des pharmaciens du Sénégal } \\ \text { PF } & \text { Planification familiale } \\ \text { PO } & \text { Partenariat de Ouagadougou } \\ \text { TPCm } & \text { Taux de prévalence contraceptive moderne } \\ \text { USAID } & \text { L'Agence des États-Unis pour le Développement International } \\ \text { VIH } & \text { Virus de l'immunodéficience humaine }\end{array}$




\section{Résumé exécutif}

\section{INTRODUCTION}

Le Gouvernement du Sénégal (GdS) s'est résolument engagé vers une politique volontariste de Planification Familiale (PF). Ainsi, en 2012, le GdS a eu à adopter un Plan d'Action National pour la PF pour la période 2012-2015 qui a été suivi par le Cadre Stratégique National de PF (CSNPF) pour la période 2016-2020. La mise en œuvre de ce CSNPF repose sur une approche multisectorielle basée sur une implication effective des différents secteurs comme le secteur privé et le niveau communautaire pour élargir l'accès à la PF volontaire en vue d'atteindre un taux de prévalence contraceptive moderne de $47 \%$ en 2020.

Dans le secteur privé, les pharmacies privées n'ont pas été activement impliquées dans l'offre de services de PF. Leur rôle est souvent limité à la fourniture de conseil, à la vente de produits PF et à la référence. Une étude exploratoire sur le rôle potentiel des pharmacies privées menée dans le cadre du Projet Evidence en 2016-2017 a révélé que les pharmacies privées sont confrontées à de nombreux obstacles à la fois d'ordre juridique et non juridique pour pouvoir jouer pleinement leur rôle dans le programme de PF au Sénégal. Toutefois il existe des opportunités qui pourraient être exploitées pour renforcer la contribution de ce secteur dans la réalisation des objectifs du programme de PF, notamment l'existence d'un réseau dense de pharmacies privées, ainsi que le fait qu'elles vendent déjà des méthodes contraceptives et offrent des conseils en PF spécifiques aux clients par rapport aux médicaments.

\section{CONCEPTION ET MÉTHODOLOGIE DE L'ÉTUDE}

En 2018, le Projet Evidence financé par l'USAID et un soutien financier de la Fondation William et Flora Hewlett ont permis au Population Council d'initier une étude pilote ayant pour but d'évaluer la faisabilité de l'offre de services de PF par le personnel des pharmacies privées dans trois (3) départements que sont Dakar, Pikine et Guédiawaye. Ses objectifs spécifiques sont: (1) évaluer les connaissances du personnel des pharmacies privées en matière de counseling en PF et de référence à trois étapes de l'étude (avant la formation, après la formation et à 1 mois et demi après la formation); (2) démontrer les connaissances et les compétences techniques requises des pharmaciens et des pharmaciens assistants pour l'offre de contraceptifs oraux et l'administration de contraceptifs injectables sur des modèles non-humains un mois et demi après la formation ; et (3) évaluer, par le biais de clientes mystères, la qualité des services offerts par le personnel des pharmacies privées en matière de counseling et de référence PF. Le protocole de recherche a reçu l'approbation éthique du Comité d'Ethique du Population Council et celle du Comité National d'Ethique pour la Recherche en Santé au Sénégal.

En partenariat avec l'Ordre des Pharmaciens du Sénégal, 17 pharmacies ont été recrutées pour l'étude et 84 personnels de pharmacies (pharmaciens, pharmaciens assistants, et agents de comptoir) ont reçu une formation en PF selon leurs qualifications. La formation a duré trois (3) jours et a été structurée comme suit : le jour 1 a été axé sur le counseling PF et la référence pour toutes les méthodes disponibles au Sénégal; le jour 2 a porté sur l'offre de contraceptif oral et les injectables; et le jour 3 a porté sur l'administration du DMPA-IM et du DMPA-SC sur des modèles. Les agents de comptoir étaient concernés seulement par la première journée de formation. Les pharmaciens et les pharmaciens assistants ont participé à tous les trois (3) jours. En plus, les pharmacies ont reçu une visite de suivi à 1 mois et demi après la formation.

Six (6) agents de collecte de données formés sur l'éthique de la recherche et les outils de l'étude, ont interviewé le personnel des pharmacies à 3 étapes pendant l'étude : une semaine avant la formation (entretien du pré- 
test, $n=84$ ), juste après la formation (entretien du post-test, $n=69$ ) et un mois et demi après la formation (entretien du suivi, $\mathrm{n}=68$ ). L'échantillon pour l'analyse a été limité aux 69 personnes disponibles pour l'enquête du post-test. Le consentement écrit, libre et éclairé, a été obtenu de chaque participant au prétest, post-test et à l'entretien de suivi. En plus, quatre (4) femmes d'âges et de milieux spécifiques ont été formées comme clientes mystères pour agir comme de nouvelles clientes de services de PF. Les clientes mystères ont utilisé chacune un des quatre (4) profiles suivant : (1) une cliente de contraceptif oral (pilule); (2) une cliente de contraceptif injectable, (3) une cliente de contraception d'urgence et ; (4) une cliente de l'implant. Après la formation, les clientes mystères ont visité durant trois (3) semaines les pharmacies privées participantes et ont rempli un questionnaire structuré évaluant le counseling reçu. Enfin, des cahiers de collecte de données ont été remis à chaque pharmacie participante pour suivre le nombre et le type de services de PF offerts.

\section{RÉSULTATS}

Basées sur l'analyse de données, les principales conclusions de l'étude sont résumées ci-dessous.

Les résultats des entretiens de post-test et des clients mystères montrent que le personnel des pharmacies avait besoin d'un soutien supplémentaire pour offrir de counselling en qualité. La plupart du personnel des pharmacies connaissait presque toutes les méthodes disponibles au Sénégal (94\%) et certaines informations clés pour les clientes, telles que les méthodes qui protègent également contre le virus de l'immunodéficience humaine et les infections sexuellement transmissibles (100\%) et le temps recommandé pour l'espacement des naissances (94\%). Concernant les questions clés de counseling et les questions visant à exclure la grossesse, les connaissances du personnel des pharmacies étaient faibles avant et même après la formation sur la base des résultats des entretiens.

L'analyse des données des clientes mystères montre qu'un soutien doit être renforcé de manière générale pour s'assurer que le personnel fourni de façon constante des services de counseling de qualité. Peu de clientes mystères (33\%) ont été informées des effets secondaires, des signes de danger (28\%) et des mesures à prendre si elles rencontrent des problèmes avec leur méthode (24\%). La plupart des clientes mystères ont été conseillées au comptoir de la pharmacie (59\%) et peu d'entre elles ont perçu une intimité auditive (47\%) ou visuelle (29\%). Cependant, dans la plupart des cas, les clientes mystères ont été interrogées sur leur expérience en matière de PF (94\%), ont été informées des autres méthodes de PF (90\%), ont été interrogées sur la méthode qu'elles préféreraient utiliser $(82 \%)$, et ont été orientées sur la façon d'utiliser la méthode de leur choix $(70 \%)$. De plus, seulement $27 \%$ des clientes mystères qui ont reçue le counseling en PF ont déclaré que leur prestataire n’a pas utilisé l'aide-mémoire.

Les entretiens de pré-test et de post-test montrent que les pharmaciens et les pharmaciens assistants ont des connaissances faibles sur les effets secondaires courants, les conditions qui limitent l'utilisation des contraceptifs oraux, et les signes avant-coureurs des contraceptifs oraux. Des résultats similaires ont été observés pour des informations clés sur les contraceptifs injectables pour lesquels les connaissances sur les effets secondaires, les conditions qui limitent son utilisation et quand une femme peut commencer l'injection étaient faibles.

Toutefois, les pharmaciens et les pharmaciens assistants ont montré qu'ils savaient bien comment expliquer aux femmes l'utilisation des contraceptifs oraux. Les connaissances étaient satisfaisantes pour les indicateurs spécifiques au DMPA-IM et au DMPA-SC, y compris le mode d'administration et les endroits où chaque méthode peut être administrée sur le corps.

\section{DÉFIS}

L'équipe de recherche a identifé les défis à relever pour permettre au personnel des pharmacies de fournir des services de PF de qualité. Premièrement, la durée de la formation de 3 jours a été un défi pour certains 
pharmaciens qui sont très occupés avec leurs activités au sein et en dehors des pharmacies. Par conséquent, des approches d'apprentissage innovantes qui respectent les horaires des pharmaciens doivent être explorées. Deuxièmement, le personnel de la pharmacie ne s'est pas conformé aux mesures de confidentialité nécessaires pour le counseling en PF et renforcé pendant la formation, alors que les pharmacies ont un espace qui pourrait assurer la confidentialité pour le counseling. Enfin, le personnel des pharmacies a besoin d'un soutien pour renforcer leur connaissances et compétences et assurer la qualité des services, ainsi l'offre de services de PF par les pharmacies privées doit être suivie et supervisée conformément aux politiques, normes et protocoles. Donc un système de suivi et de supervision adéquat est nécessaire.

\section{CONCLUSION ET RECOMMANDATIONS}

Le personnel des pharmacies constitue pour les populations une source potentielle de services de counseling, de contraceptifs oraux et de services d'injectables, mais un appui supplémentaire est nécessaire. Toutefois, des efforts méritent d'être consentis pour respecter les normes de la qualité requises avec un accent particulier sur le dépistage des clientes pour s'assurer qu'elles sont éligibles sur le plan médical et sur la fourniture aux clientes d'informations complètes sur les effets secondaires.

Sur la base de ces constats, les recommandations suivantes ont été formulées :

- Explorer les voies et moyens pour la mise en place d'un environnement juridique favorable et la mise en oeuvre d'interventions qui s'avèrent nécessaires avec les pharmacies privées sur l'offre d'injectables aux clientes de PF volontaire.

- Explorer de nouvelles approches de formation telles que les formations sur sites (au sein des pharmacies) ou des formations durant les week-ends pour assurer le temps suffisant (un minimum de 32 heures) à consacrer à la pratique et la concentration sur le respect des procédures.

- Explorer les délégués médicaux comme un moyen de renforcer la connaissance de la PF avec les pharmaciens privés.

- Encourager les pharmaciens à aménager un espace adéquat pour le counseling en PF et explorer les interventions du changement de comportement pour aider le personnel des pharmacies privées à fournir des services dans un milieu privé et non au comptoir.

- Mettre en place des stratégies permettant aux pharmaciens d'acquérir des compétences additionnelles telles que la formation continue et/ou les supervisions formatives.

- Institutionaliser un système de supervision de l'assurance qualité avec l'Ordre des Pharmaciens directement impliqué dans l'évaluation régulière de la qualité dans l'offre de services de PF.

- Sensibiliser davantage les pharmaciens sur les enjeux de leur implication dans l'offre de services de PF et sur l'importance d'utiliser les outils d'aide au travail pour assurer la qualité et se conformer aux normes et protocoles. 


\section{Introduction}

Au cours de la dernière décennie, le Gouvernement du Sénégal (GdS), à l'instar des pays du Partenariat de Ouagadougou (PO), ${ }^{1}$ s'est résolument engagé vers une politique volontariste de Planification Familiale (PF). Ainsi, en 2012, pour matérialiser cet engagement, le GdS a eu à adopter un Plan d'Action National pour la PF pour la période 2012-2015. Les objectifs de ce plan étaient de relever le Taux de Prévalence Contraceptive moderne (TPCm) de 12\% en 2012 à $27 \%$ en 2015 puis à 45\% en 2020 (Ministère de la Santé et de l'Action sociale [MSAS], Plan d'Action National pour la PF, 2012). Cette volonté de repositionner la PF s'inscrivait, d'une part, dans les axes stratégiques du Plan Sénégal Emergent qui la définit comme une des interventions majeures pour réduire les taux élevés de mortalité maternelle, néonatale et infantile et comme stratégie prioritaire devant permettre d'assurer la transition démographique en vue d'atteindre le dividende démographique ; d'autre part, ce soutien des autorités publiques en faveur de la PF s'inscrivait dans le cadre des engagements pris à travers les initiatives sous-régionales, notamment ceux du $\mathrm{PO}^{2}$ et internationales, notamment la déclaration du sommet de Londres (le FP2020) qui vise à recruter d'ici 2020 plus de 120 millions nouvelles utilisatrices additionnelles de méthodes modernes de $\mathrm{PF}^{3}$ Par ailleurs, ces options sont en conformité avec les engagements pris par les pays membres du système des Nations Unis par rapport aux Objectifs du Millénaire pour le Développement qui ont été mis à jour récemment à travers l'adoption des Objectifs du Développement Durable. En 2016, le GdS, à travers l'adoption du nouveau Cadre Stratégique National de PF (CSNPF) pour la période 2016-2020 a renouvelé son engagement d'un soutien plus effectif en faveur de la PF. La mise en œuvre de ce CSNPF repose sur une approche multisectorielle basée sur une implication effective des différents secteurs, particulièrement du secteur privé, y compris les pharmacies, et du niveau communautaire dont les contributions attendues à la réalisation du TPCm sont respectivement de $28 \%$ et 47\% (CSNPF, 2016-2020). Le nouveau CSNPF a réaffirmé cette volonté de renforcer le rôle du secteur privé dans l'offre de services de PF. Dans ce nouveau CSNPF, il est attendu du secteur privé une contribution de $28 \%$ à la réalisation de l'objectif de $45 \%$ de Taux de Prévalence Contraceptive en 2020. De plus, lors du dernier sommet de Londres de 2017 sur la PF, le Sénégal, à l'instar des autres pays du Partenariat de Ouagadougou, a réitéré son engagement à renforcer l'offre de service dans le secteur privé. Parmi les mesures devant accroitre le rôle du secteur privé, figurent la révision des textes juridiques et réglementaires ainsi que le renforcement des compétences du personnel des pharmacies privées en technologie contraceptive.

Enfin, l'adhésion du GdS à l'initiative du Global Financing Facility en faveur d'une mobilisation plus accrue des fonds domestiques pour la mise en œuvre des programmes de santé maternelle néonatale et infantile ainsi que des programmes de PF confirme cette volonté des autorités publiques de renforcer et pérenniser les interventions en faveur de la PF.

Toutefois, si ces engagements ont permis d'enregistrer des progrès notables en termes d'augmentation du TPCm (l'indicateur passant de 16\% en 2012 à 23\% en 2015 puis à 26\% en 2016), il convient cependant de noter que les résultats ont été surtout réalisés au niveau du secteur public et au niveau communautaire ; le secteur privé pharmaceutique, malgré le potentiel dont il dispose ne joue pas encore un rôle important dans l'offre de services de PF.

${ }^{1}$ Le Partenariat de Ouagadougou crée en 2011 sous le thème « Urgence d'agir » est constitué de neuf (9) pays francophones de l'afrique de l'ouest dont le Bénin, le Burkina Faso, la Côte d'Ivoire, la Guinée, le Mali, la Mauritanie, le Niger, le Sénégal et le Togo, et avait pour ambition d’atteindre 1 million de femmes supplémentaires pour la période 2012-2015.

${ }^{2}$ Le Partenariat de Ouagadougou a adopté la «phase d'accélération » pour la période 2016-2020 pour recruter 2200000 nouvelles utilisatrices de PF supplémentaires d'ici 2020.

${ }^{3}$ FP2020 : FP2020 est un mouvement mondial qui contribue notamment à « Chaque Femme, Chaque Enfant », la campagne du Secrétariat Général des Nations Unies dédiée à la santé maternelle et infantile. FP2020 fédère plus de 90 organisations de la société civile (OSC), partenaires techniques et financiers, gouvernements, entreprises privées et membres de la communauté scientifique de 69 pays en développement autour d'un objectif commun : renforcer la demande et l'offre en PF pour atteindre 120 millions de nouvelles utilisatrices de contraception moderne dans ces pays d'ici 2020. 
En effet, les pharmacies privées n'ont pas été jusqu'à présent activement impliquées dans l'offre de services de PF. Un premier obstacle est que la législation du Sénégal précise que l'exploitation des pharmacies privées « est incompatible avec l'exercice d'une autre profession, notamment celle de médecin, vétérinaire, sage-femme ou dentiste » (Code de Santé Publique, 1954). La législation empêche aussi les pharmacies privées d'effectuer des actes médicaux telles que les injections. Les obstacles non juridiques incluaient le manque de formation en PF, la faible connaissance des méthodes contraceptives et l'inadéquation des infrastructures, notamment l'absence d'un espace approprié pour offrir des services de counseling en PF pour certaines pharmacies (Mbow et al., 2017). Par ailleurs, la faible collaboration entre le secteur privé et les districts sanitaires ne milite pas en faveur d'une implication effective des pharmacies privées dans cette dispensation des services de PF. Une étude exploratoire sur le rôle potentiel des pharmacies privées dans l'offre de services de PF au Sénégal, réalisée en 2016 par le Population Council en collaboration avec la Direction de la santé de la reproduction et de la survie de l'enfant ${ }^{4}$, avec le soutien financier de l'Agence des États-Unis pour le Développement International (USAID) dans le cadre du Projet Evidence et de la Fondation William et Flora Hewlett, a démontré que la perspective de mieux impliquer les pharmacies privées dans l'offre de services de PF était acceptable par les parties prenantes du programme de PF, et que celles-ci pouvaient jouer un rôle plus important dans l'amélioration de l'accès aux services de PF si les obstacles étaient levés et si les compétences en technologie contraceptive du personnel des pharmacies étaient renforcées (Mbow et al., 2017). Cette étude exploratoire a révélé que les pharmacies privées sont confrontées à de nombreux obstacles à la fois d'ordre juridique et non juridique pour pouvoir jouer pleinement leur rôle dans le programme de PF au Sénégal.

Toutefois, tout en signalant ces faiblesses, les résultats de l'étude exploratoire ont révélé quelques opportunités qui pouvaient être exploitées pour renforcer la contribution de ce secteur dans la réalisation des objectifs du programme de PF. Parmi ces opportunités on peut noter l'existence d'un réseau dense de pharmacies privées avec une bonne couverture géographique au niveau national. Les pharmacies privées font déjà partie du système de santé puisqu'elles vendent des méthodes contraceptives - les préservatifs, les colliers du cycle, les contraceptives oraux (y compris contraception d'urgence), les injectables, les implants et dans quelques pharmacies les DIU ${ }^{5}$ - et offrent des conseils spécifiques aux clients par rapport aux médicaments. Des études ont montré que la quasi-totalité des pharmacies vendait au moins une forme de contraceptif (Agence pour la Promotion des Activités de Population, 2016) et que 9\% des femmes mariées utilisant actuellement une méthode moderne avaient reçu leur dernière méthode contraceptive dans une pharmacie privée (Agence nationale de la statistique et de la démographie et ICF, 2016). En 2009, les pharmacies privées constituaient la principale source d'approvisionnement pour la contraception d'urgence, un produit pouvant désormais être vendu sans ordonnance (Diop, 2009). D'autres études ont révélé que, malgré les restrictions légales, certaines pharmacies privées offraient des services additionnels de PF. Une étude menée par le MSAS et FHI360 auprès de 225 pharmacies a révélé qu'environ 6\% d'entre elles offraient des contraceptifs oraux, 4\% fournissaient des contraceptifs injectables aux clientes sans ordonnance, et 15\% administraient les injectables sur place malgré les régulations juridiques (Fall, 2015).

Dans le cadre des programmes de marketing social, plusieurs pharmaciens et agents de comptoir avaient eu à bénéficier d'orientation sur la PF et récemment des initiatives pilotes et des programmes de formations menées par des organisations non-gouvernementale telles que l'Agence pour le Développement du Marketing Social et Marie Stopes International ont démontré que les pharmacies privées présentaient un potentiel réel pour contribuer à l'atteinte des objectifs du programme de PF (Initiative Sénégalaise de Santé Urbaine, 2014). La formation du personnel des dépôts et officines pharmaceutiques a été identifiée comme l'une des "pratiques à haut impact » les plus prometteuses dans le domaine de la PF (PHI, 2013).

${ }^{4}$ Cette Direction est devenue en 2017 la Direction de la Santé de la Mère et de l'Enfant.

${ }^{5}$ Pour la vente des contraceptifs oraux (exceptée la contraception d'urgence), injectables, implants et DIU, une pharmacie peut vendre ces méthodes aux clientes sur présentation d'une ordonnance. La cliente peut alors se rendre chez un médecin privé pour l'administration ou l'insertion. 


\section{Contexte de la mise en œuvre de l'étude pilote}

Le Population Council à travers le Projet Evidence a collaboré avec la DSME pour la conception d'une étude pilote sur l'offre des services de PF par les pharmacies privées, s'inscrivant dans les orientations stratégiques du nouveau CSNPF pour la période 2016-2020 adopté en juin 2016 qui consacre, à côté des interventions des pouvoirs publics, un rang de priorité à l'implication des acteurs du secteur privé et du niveau communautaire pour une meilleure contribution à l'atteinte des objectifs de santé publique que le MSAS s'est fixé. Cette volonté politique a commencé à se matérialiser depuis l'adoption du premier Plan d'Action National pour la Planification Familiale à travers la mise en œuvre d'interventions ciblées au niveau de ce secteur. En plus d'une opérationnalisation de l'approche inclusive et multisectorielle définie par le CSNPF, la présente étude pilote est la suite logique d'étude exploratoire réalisée en 2015.

A travers cette étude pilote, la DSME veut s'appuyer sur des preuves pour renforcer son plaidoyer en faveur d'une plus grande implication des pharmacies privées dans le programme de PF. Sur la base des résultats de l'étude exploratoire en 2016-2017, la DSME et des partenaires clés travaillant avec le secteur privé ont identifié un besoin pour des évidences supplémentaires sur la faisabilité de l'offre des services en PF par les pharmacies privées, spécifiquement le counseling en PF, l'offre de contraceptifs oraux et l'administration de contraceptifs injectables, et les références aux centres de santé publics ou privés pour toutes les méthodes disponibles au Sénégal. Comme décrit ci-dessus, les services de PF que les pharmacies privées sont autorisées à offrir se limitent à la vente de produits contraceptifs sur la base d'une ordonnance pour certains produits classé sur les listes I et II de la nomenclature des médicaments, à la vente de préservatifs, de contraceptifs d'urgence et de collier sans une ordonnance, à l'offre de conseils, et la référence des client(es) en cas de besoin.

Donc en 2017, le Population Council à travers le Projet Evidence et la DSME ont développé une étude pilote de la science de la mise en œuvre dans le cadre de laquelle la pharmacie privée en tant qu'unité - les pharmaciens, les pharmaciens assistants et les agents du comptoir — serait formée sur le counseling en PF, la sélection des femmes intéressées aux contraceptifs oraux et injectables sur la base des critères d'éligibilité médicale, l'offre de contraceptifs oraux aux clientes et l'administration des contraceptifs injectables aux clientes. Le personnel des pharmacies privées recevrait également une formation sur la référence dans les situations suivantes : (1) les personnes intéressées par l'implant, le DIU, la vasectomie et la stérilisation féminine ; (2) les personnes qui entrent dans les catégories 2 à 4 selon les critères d'éligibilité médicales de l'OMS pour la méthode souhaitée ; et (3) les personnes qui préfèrent recevoir leur méthode d'un prestataire public ou privé. L'étude avait été initialement conçue pour que tous les personnels reçoivent la même formation et fournissent les mêmes services de PF pendant toute la durée de l'étude.

En outre, au cours de la phase préparatoire de l'étude, l'équipe de recherche a travaillé avec des formateurs de la DSME et des formateurs indépendants pour élaborer un curriculum de formation basée sur des matériels d'apprentissage déjà validés et utilisés au niveau mondial et au Sénégal. Le curriculum a également été conçu sur le modèle de la formation menée avec succès auprès des vendeurs de médicaments dans le secteur privé au Nigéria. Les formateurs ont revu le contenu pour s'assurer de son exactitude et de sa conformité avec les normes et protocoles du programme de PF du Sénégal.

\section{RÉVISIONS DU PROTOCOLE}

En raison de l'environnement juridique au Sénégal qui ne permet pas aux pharmacies privées de pratiquer l'injection, et du fait de l'absence ou insuffisance de coordination entre ces pharmacies privées et les districts sanitaires, le protocole initial de l'étude a fait l'objet de révisions. Premièrement, les agents de comptoir ont 
été exclus des séances de formation sur les contraceptifs oraux et injectables sur demande du Comité national d'éthique pour la recherche en santé (CNERS) parce que certaines études ont montré qu'ils n'ont pas tous reçu une formation médicale à la base. La pharmacie, par conséquent, n'était plus formée en tant qu'unité pour les trois services de PF. Les agents du comptoir n'ont participé qu'à la séance sur le counseling et l'orientation en matière de PF, tandis que les pharmaciens et les pharmaciens assistants ont participé aux séances sur le counseling en matière de PF, l'offre de contraceptifs oraux et l'administration des contraceptifs injectables. Bien que des études antérieures menées dans d'autres pays aient formé du personnel du secteur privé ayant des qualifications similaires à ces compétences et que des agents de santé communautaire aient été formés pour l'offre de pilules et l'administration des injectables au Sénégal dans le cadre de programmes pilotes, les agents de comptoir n'ont pas été autorisés à être formés pour l'offre de ces services dans le cadre de cette étude.

Deuxièmement, puisque le suivi et la supervision de leurs activités ne sont pas intégrés dans la supervision de routine au niveau des districts dont elles relèvent, le comité d'éthique s'est inquiété de savoir qui serait responsable si une femme souffrait d'un effet indésirable en conséquence d'un contraceptif injectable reçu d'une pharmacie participante. Par conséquent, les pharmaciens et les assistants pharmaciens ont reçu une formation sur l'administration des injectables sur les modèles, mais il leur a été interdit d'administrer des contraceptifs injectables aux femmes qui pourraient demander ces services auprès de leur pharmacie pendant l'étude pilote. Après les formations, les pharmaciens et les assistants pharmaciens ont fourni le counseling en PF et offert les contraceptifs oraux directement aux clientes (sans ordonnance), et les agents du comptoir ont fourni uniquement le counseling en PF.

\section{RÉVISION DES OUTILS ET DE LA DURÉE DE LA FORMATION}

Pendant que l'équipe de recherche attendait l'approbation éthique, les partenaires clés de la mise en œuvre de l'étude, notamment l'OPS et les formatrices du MSAS, ont examiné le curriculum de formation pour s'assurer de son applicabilité aux pharmacies privées. En raison des engagements des pharmaciens privés et de leurs horaires chargés, l'OPS a identifié la durée de la formation comme pouvant être un obstacle pour la pleine participation des pharmaciens. Ainsi, le temps alloué à la formation a été réduit de 32 à 15 heures mais avec la même quantité de contenu d'apprentissage.

\section{CONSÉQUENCES}

Les révisions apportées au protocole pour répondre aux préoccupations du comité d'éthique ont retardé le processus d'approbation éthique pour l'étude au Sénégal, réduisant ainsi le temps disponible pour la mise en œuvre de l'étude de 10 à 4 mois. Ces retards ont non seulement réduit la période de suivi prévue de 5 mois après la formation à environ 6 semaines, mais n'a pas permis à l'équipe de recherche de faire les adaptations nécessaires pour l'intervention. De telles adaptations auraient inclus un coaching continu et une pratique simulée pour les pharmaciens et les pharmaciens adjoints après la formation, et la révision du format de la formation pour l'adapter aux horaires des pharmaciens.

Pour ces raisons, les résultats présentés dans ce rapport ne donnent pas une image complète du potentiel des pharmacies privées à l'offre des services de PF. Cependant, cette expérience fournie des leçons importantes pour la DSME et d'autres partenaires intéressés à travailler avec les pharmacies privées dans le cadre de la PF.

Les sections ci-dessous décrivent les méthodes, les résultats et les implications pour la conception finale de l'étude. Même avec les défis de mise en œuvre mentionnés ci-dessus, les résultats issus de cette étude avec les pharmacies privées contribueront aux discussions en cours sur les politiques pour l'implication des pharmacies privées dans l'offre de services de PF. En outre, le Sénégal étant un acteur clé du PO, les résultats de l'étude avec les leçons apprises de la mise en œuvre de l'étude contribueront au débat régional sur les stratégies visant à accroitre l'accès et l'utilisation de la PF en Afrique de l'Ouest francophone. 


\section{Objectifs de l'étude}

L'étude avait pour but d'évaluer la faisabilité de l'offre de services de PF par le personnel des pharmacies privées.

Ses objectifs spécifiques sont les suivants :

1. évaluer les connaissances du personnel des pharmacies privées (pharmaciens, pharmaciens assistants et agents de comptoir) en matière de counseling en PF et de la référence à trois étapes de l'étude (avant la formation, après la formation et à 1 mois et demi après la formation) ;

2. démontrer les connaissances et les compétences techniques requises des pharmaciens et des pharmaciens assistants pour l'offre de contraceptifs oraux et l'administration de contraceptifs injectables (par voies intramusculaire (ex : Depo-Provera) et sous-cutanée (ex : Securil Press) sur des modèles non-humains (1 mois et demi après la formation), ceci en référence aux protocoles en vigueur); et

3. évaluer, par le biais de clientes mystères, la qualité des services offerts par le personnel des pharmacies privées en matière de counseling et de référence PF. 


\section{Méthodologie}

\section{PROCÉDÉ DE RECHERCHE}

Dans le cadre de cette étude, un procédé pré et post sans groupe de contrôle a été utilisé, avec : i) trois enquêtes : une enquête pré-test, une enquête post-test et une enquête de suivi à un mois et demi; ii) une intervention axée sur le renforcement des capacités du personnel des pharmacie privées en counseling et la référence, et sur l'offre de la PF pour les pharmaciens et pharmaciens assistants ; iii) une enquête cliente mystère et; iv) une visite de suivi à 1 mois et demi après la formation. Les données ont été ainsi collectées à plusieurs étapes pour fournir des évidences sur la capacité des pharmacies privées dans l'offre de counseling et de quelques méthodes de PF telles que la pilule et les injectables.

\section{SITES D'ÉTUDE}

L'étude s'est déroulée dans la région de Dakar au niveau des trois (3) départements que sont Dakar, Pikine et Guédiawaye. Le choix de la région de Dakar est justifié non seulement par le fait que cette zone compte le plus grand nombre de pharmacies, environ 500 pharmacies, dans le pays (Mbow, 2017), mais aussi par la nécessité de consolider les évidences tirées de l'étude exploratoire de 2015 financée par l'USAID dans le cadre du Projet Evidence et la Fondation William et Flora Hewlett ainsi que celles d'études réalisées dans la région par d'autres partenaires.

La base de l'échantillonnage de 50 pharmacies privées a été fournie par l'Ordre des Pharmaciens du Sénégal (OPS) avec la collaboration du Syndicat des pharmaciens. Ces deux organisations sont des organes de représentation des pharmacies privées qui ont aidé à identifier les pharmacies qui répondaient aux critères d'éligibilité ${ }^{6}$ établies pour cette étude, notamment :

- l'engagement du pharmacien titulaire à participer à toute la formation ;

- l'engagement du pharmacien titulaire à envoyer le personnel de la pharmacie (pharmaciens assistants et agents de comptoir) à la formation ;

- l'aptitude des agents de comptoir à parler et lire en français ; et

- la disponibilité d'un espace au sein de la pharmacie qui puisse assurer l'offre de services de PF.

Ensuite les pharmacies sélectionnées au hasard dans l'étude ont été contactées pour confirmer leur éligibilité. Pendant ces visites, la lettre d'introduction élaborée par l'OPS et celles d'approbation nécessaires ont été transmises aux pharmacies privées enrôlées, de même, les suggestions de dates et lieux des sessions de formation leur ont été soumises. Vingt (20) pharmacies ont été retenues et sur ces 20 pharmacies ciblées, trois (3) d'entre elles n'ont pas pu participer à l'étude en raison de la non disponibilité du pharmacien titulaire ou d'une partie du personnel à un moment quelconque de la mise en œuvre de l'étude (pré-test, formation ou post-test). Finalement, 17 pharmacies ont été enrôlées, 9 à Dakar, 4 à Guédiawaye, et 4 à Pikine.

\section{ACTIVITÉS D'INTERVENTION}

\section{Activités Préparatoires}

Avant la collecte des données et la formation en PF du personnel des pharmacies privées, une série d'activités préparatoires ont été menées : (1) les visites auprès des partenaires clés ; (2) le développement d'un curriculum de la formation en PF ; (3) les visites des pharmacies présélectionnées; et (4) la création d'une affiche de la demande.

${ }^{6}$ Puisque les pharmacies privées au Sénégal vendent au moins une méthode contraceptive, la vente de contraceptifs n'était pas un critère d'éligibilité pour cette étude. 
Premièrement, pour encourager l'adhésion des parties prenantes au début de l'étude et une bonne appropriation des résultats, des visites ont été effectuées auprès des parties prenantes clés — la plupart membres du comité technique de PF et ayant un intérêt particulier sur la question des pharmacies privées — pour les informer de l'étude et recueillir leurs suggestions pour orienter la sélection des pharmacies à enrôler, élaborer l'agenda et les matériaux de formation, et définir la durée, la période et le nombre de sessions de formation à organiser. Il s'agit notamment de : représentants du MSAS, USAID Sénégal, Unité de coordination du PO, Direction de la Pharmacie et du Médicament, OPS, Syndicat des Pharmaciens Privés du Sénégal, Marie Stopes International, PATH, SHOPS Plus et l'Agence pour le Développement du Marketing Social.

Le Projet Evidence a collecté du matériel d'apprentissage sur le counseling de la PF, l'offre de contraceptif oral et de contraceptif injectables utilisé au Sénégal et mondialement. Ces matériaux ont été révisés, consolidés et adaptés pour une formation de trois (3) jours du personnel des pharmacies privées. Après la création du curriculum consolidé, l'équipe des facilitateurs/formateurs, y compris les représentants du MSAS et de l'OPS ont été invités durant trois (3) jours au cours d'une rencontre à examiner le curriculum ainsi que les matériaux de référence pour la formation en vue de leur finalisation. Cette équipe a également contribué à documenter les changements apportés sur les matériaux pour une éventuelle mise à l'échelle au niveau national. Cette démarche a permis de s'assurer que la formation tient compte de la situation spécifique des pharmacies privées au Sénégal.

Avant la formation en PF, l'équipe de recherche a visité les pharmacies sélectionnées. Ces visites ont été également l'occasion pour les pharmaciens titulaires d'identifier, qui parmi le personnel serait éligible, intéressé et disponible pour participer à la formation et pour l'équipe de recherche de vérifier que les pharmacies possédaient véritablement un espace pour assurer l'offre de services. L'équipe de recherche a également assuré la coordination avec les intervenants appropriés afin de s'assurer que les pharmacies enrôlées auront suffisamment de stock de produits contraceptifs pendant toute la durée de l'étude. En plus, les pharmacies privées ont été informées de l'utilisation des clientes mystères dans l'étude.

Enfin, en vue de générer la demande de services au niveau des pharmacies privées pendant l'étude, une affiche a été élaborée et finalisée avec l'aide des participants à l'étude (Annexe 1). Les commodités de PF n'ont pas été fournies aux pharmacies privées dans le cadre de cette étude. Les médicaments ont été achetés par les canaux d'approvisionnement existants.

\section{L'intervention : formation en PF pour le personnel des pharmacies privées}

Pour s'assurer que la pharmacie privée puisse fournir des services de PF aux clientes, l'ensemble du personnel de la pharmacie (c.a.d. pharmacien, pharmacien assistant, et agents de comptoir) a reçu une formation en PF selon leurs qualifications. La formation a duré trois (3) jours et a été structurée comme suit : le jour 1 a été consacré au counseling en PF et la référence ${ }^{7}$ pour toutes les méthodes disponibles au Sénégal ; le jour 2 a porté sur l'offre des contraceptifs oraux et les injectables; et le jour 3 a porté sur l'administration du DMPA-IM et du DMPA-SC sur des modèles. Les agents de comptoir étaient concernés seulement par la première journée de formation. Les pharmaciens et les pharmaciens assistants ont participé à tous les trois jours. ${ }^{8}$ La formation du jour 2 a porté sur des aspects plus approfondis concernant le counseling des femmes qui utilisent les contraceptifs oraux et les injectables, notamment les rendez-vous relatifs aux visites de suivi pour le réapprovisionnement ou la réinjection, la prise en charge des effets indésirables ainsi que la conduite à tenir si un tel événement indésirable se produisait. Le jour 3, les pharmaciens et les pharmaciens assistants ont été formés sur l'administration de DMPA-IM et DMPA-SC sur des modèles. Enfin, la formation pour tous les personnels a intégré la gestion des données, notamment le remplissage du cahier de collecte.

\footnotetext{
${ }^{7}$ Tout le personnel des pharmacies privées a été formé sur la référence des clientes qui auraient choisi un contraceptif injectable ou une méthode à longue durée d'action, qui auraient une prédisposition qui pourrait les rendre inéligibles pour le contraceptif oral, ou pour des clientes dont on ne peut déterminer l'état de grossesse.

${ }^{8}$ Pour des contraintes liées aux exigeances du comité éthique l'administration des injectbales a été effectuée sur des modèles et non sur des cas réels comme initialement prévu.
} 
La formation en counseling a été basée sur la stratégie de Counseling équilibré Plus [Balanced Counseling Strategy Plus (BCS+)] développé par le Population Council. La formation sur l'offre des contraceptifs oraux et l'administration des injectables, qui n'a concerné que les pharmaciens et pharmaciens assistants, a utilisé le matériel de formation en PF standardisé au niveau national y compris les matériaux de formation développés par PATH sur les contraceptifs injectables, notamment sur le DMPA-IM et le DMPA-SC sur des modèles (des oranges). La formation a été animée par des formateurs expérimentés provenant de la DSME, de l'OPS, et des formateurs indépendants.

Des méthodes andragogiques telles que les démonstrations, les jeux de rôles, les exercices pratiques et les discussions de groupe ont été utilisées pour s'assurer de l'acquisition et de l'application par les participants des connaissances et des compétences nécessaires à l'exécution de ces tâches.

A la fin de la formation, les pharmacies privées ont reçu les outils d'aide au travail, y compris la boîte à outils BCS+, et les listes de contrôle de FHI 360 pour dépister les nouvelles utilisatrices de contraceptifs injectables et de contraceptifs oraux combinés, le guide de référence sur les critères d'éligibilité médicale de l'Organisation Mondiale de la Santé (OMS), les outils d'aide au travail pour la gestion des oublis lors de l'utilisation des pilules, ainsi que de cahiers de collecte des données dans la perspective de l'intégration des données dans le système national d'information sanitaire.

\section{Visites de Suivi Auprès des Pharmacies Enrôlées}

Les formateurs disponibles se sont réparti les pharmacies privées à visiter et ont procédé à une visite de suivi à 1 mois et demi après la formation. Durant cette visite de suivi, ils ont administré les guides d'observation pour évaluer les compétences du personnel des pharmacies sur l'offre de contraceptifs oraux et l'administration du DMPA-IM et DMPA-SC sur les modèles. En outre, les formateurs ont vérifié lors de leurs visites la qualité du remplissage du cahier de collecte pour les clientes reçues.

Cette visite de suivi a donné également au personnel des pharmacies privées l'opportunité de discuter directement avec les formateurs de leurs expériences, notamment de ce qui a bien marché mais aussi des difficultés rencontrées. Ce fut également l'occasion pour les formateurs de voir les progrès de l'étude et d'offrir au personnel des pharmacies un soutien et des feedbacks.

\section{COLLECTE DES DONNÉES}

\section{Les Entretiens Individuels Structurés}

Trois (3) enquêtes structurées (enquêtes du pré-test, post-test et du suivi à 1 mois et demi) ont été utilisées pour évaluer les connaissances du personnel des pharmacies avant la formation, juste après et à 1 mois et demi après la formation. Les outils de collecte de données ont été élaborés par l'équipe de recherche et adaptés à partir de ceux de l'étude réalisée par le Projet Evidence sur l'examen et le renforcement du rôle des vendeurs de médicaments brevetés dans l'offre de contraceptifs injectables au Nigéria (Ishaku, 2018). En plus, l'équipe de recherche a utilisé les aides au travail d'offre du contraceptif oral et du counseling en PF pour élaborer les questions sur ces sujets. Les outils ont été revus en équipe pour être davantage peaufinés pendant la phase de préparation de cette étude. Ils ont été testés avant le démarrage de la collecte.

Le questionnaire pré-test a inclut des questions sur les caractéristiques soci-démographiques des participants, les types de services de PF que la pharmacie offre, et sur les connaissances du counseling, les contraceptifs oraux et les contraceptifs injectables. L'objet du pré-test a été d'évaluer la connaissance des participants et de comprendre les types de services fournis avant la formation. Le questionnaire post-test, administré juste après la formation, a inclu les mêmes questions de connaissance du pré-test pour évaluer les changements de connaissance. 
Le questionnaire du suivi a inclus les mêmes questions des connaissances du pré-test et du post-test, mais a aussi inclut des questions supplémentaires sur la gestion des données. En outre, l'enquête de suivi comprenait des questions sur les expériences du personnel des pharmacies en matière d'offre de services de PF. Il s'agissait aussi de voir si la formation les a préparés à conduire le counseling en PF, à offrir des contraceptifs oraux et des injectables, et s'ils ont besoin d'acquérir des compétences additionnelles et aussi des recommandations pour étendre cette formation au personnel des autres pharmacies privées.

La collecte des données auprès du personnel des pharmacies privées participant à l'étude a été effectuée par six (6) agents de collecte de données formés durant quatre (4) jours. Le pré-test a été réalisé une semaine avant la formation du personnel des pharmacies sur leur lieu de travail avec leur consentement. Par ailleurs, des enquêtes de post-test ont été menées aussitôt après la formation : à l'issue du 3ème jour pour les pharmaciens et pharmaciens assistants, et le jour suivant la formation sur le counseling pour les agents de comptoir. Les enquêtes de suivi ont été menées à 1 mois et demi après la formation sur le lieu de travail des participants.

\section{Visites des Clientes Mystères}

Quatre (4) femmes d'âges et de milieux spécifiques ont été recrutées en tant que clientes mystères faisant partie de l'équipe d'étude. Elles ont été sélectionnées pour représenter la clientèle type (Fitzpatrick, 2017) des pharmacies privées de Dakar.

Les clientes mystères ont participé à une formation de quatre (4) jours, qui ont porté sur le but de l'étude, leur rôle en tant que cliente mystère, la mémorisation de leur profil de client pour une demande de services de PF, la pratique des séances de counseling, la manière d'éviter les procédures invasives, comment se sortir d'une situation non-désirable et comment remplir le formulaire structuré. La méthodologie de collecte d'informations a été systématiquement expliquée aux clientes mystères avec comme support un algorithme décrivant le processus de collecte. La tenue vestimentaire et le langage appropriés ont été discutés pendant leur formation afin de s'assurer que ces dernières sont représentatives de la clientèle de la communauté.

Après la formation du personnel des pharmacies privées, les clientes mystères ont visité durant trois (3) semaines les pharmacies dont le pharmacien titulaire a complété la formation sous leur profil de client PF. Lors des visites, les clientes mystères ont agi comme de nouvelles clientes de services de PF. Elles ont prétendu être de vraies clientes de services de PF et ont utilisé chacune son propre profil démographique et ont reçu l'un des quatre (4) profils de PF développés par l'équipe de recherche, y compris la préférence pour la méthode contraceptive, que sont les suivants :

- cliente recherchant une méthode contraceptive orale (pilule);

- cliente recherchant une méthode injectable ;

- cliente recherchant une méthode contraceptive d'urgence ; et

- cliente recherchant un implant.

Les clientes mystères ont rempli un questionnaire structuré évaluant le counseling reçu au niveau de la pharmacie privée dans les 90 minutes suivant leur visite et avant d'effectuer les autres visites. Chaque pharmacie a reçu environ 3 visites de clientes mystères.

\section{Recueil des Données à Travers les Cahiers de Collecte}

Des cahiers de collecte de données élaborés par l'équipe de recherche avec l'aide des formateurs ont été remis à chaque pharmacie participante pour suivre le nombre et le type de services de PF offerts par les pharmacies privées. 


\section{ASPECTS ÉTHIQUES}

\section{Approbation Éthique}

Le protocole de recherche a reçu l'approbation éthique du Comité d'Ethique du Population Council et celle du Comité National d'Ethique pour la Recherche en Santé du MSAS, ainsi qu'une Autorisation Administrative de la Direction de la Planification, de la Recherche et des Statistiques du MSAS.

\section{Consentement Éclairé}

Le consentement écrit, libre et éclairé, a été obtenu de chaque participant au prétest, post-test et à l'enquête de suivi. Les informations et exigences liées à la participation à l'étude ont été lues par les agents de collecte aux participants qui ont exprimé leur accord et signé le formulaire de consentement éclairé.

\section{Confidentialité et Anonymat}

Plusieurs mesures ont été prises afin de garantir la confidentialité et l'anonymat des informations reçuellies lors des enquêtes prétest, post-test et de suivi : les entretiens ont été réalisées par des agents de collecte expérimentés et qui ont reçu une formation appropriée pour garantir l'intimité, la confidentialité et l'anonymat de tous les participants à l'étude. Aucune information d'identification n'a été enregistrée sur le questionnaire et des codes d'identification ont été utilisés afin que l'on ne puisse établir un lien entre les réponses personnelles et une personne en particulier. Les entretiens ont eu lieu dans des endroits le plus privé possible au niveau des pharmacies. Les ordinateurs utilisés pour la saisie de données sont protégés par des mots de passe ; les notes et les questionnaires sont gardés dans des placards verrouillés au bureau du Population Council. Enfin, les cahiers de collecte n'ont inclus aucune information identifiable sur le client ou le personnel de la pharmacie qui a remplit le formulaire.

Chaque cliente mystère a signé un formulaire de non-divulgation des informations collectées.

\section{Compensation}

Le personnel des pharmacies n'a reçu aucune compensation financière pour sa participation à cette étude. Seuls leurs frais de transport ont été remboursés.

\section{GESTION ET ANALYSE DES DONNÉES}

\section{Saisie des Données}

Les enquêtes ont été enregistrées sur papier et la saisie des données a été faite sous le logiciel CsPro. Lors de l'élaboration des maquettes de saisie, des contrôles de cohérence ont été intégrées pour minimiser les erreurs liées à la saisie. Un agent de saisie expérimenté a été orienté sur le remplissage de la maquette de saisie. Les réponses aux questions ouvertes ont été passées en revue et recodées si nécessaire. Après la saisie, l'équipe de recherche a fait un contrôle de qualité des réponses à travers la génération de fréquences simples des variables

et les erreurs de cohérence ont été corrigées. Les données ont été ensuite transférées sur SPSS et STATA pour les besoins d'exploitation et d'analyse.

\section{Analyse des Données}

Les analyse descriptives ont été utilisées pour l'analyse des données de pré-tests, de post-tests, et des clientes mystères. 


\section{Résultats}

\section{PROFIL DU PERSONNEL DE PHARMACIE}

Au total, 84 personnels de 17 pharmacies privées ont été interviewés lors de l'enquête pré-test, mais 15 d'entre eux n'étaient pas disponibles pour l'enquête post-test, ce qui portait l'échantillon final à 69. Le Tableau 1 montre les caractéristiques du personnel des pharmacies à l'enquête du pré-test. Un peu plus de la moitié du personnel de pharmacies était des hommes (54\%), avait moins de 40 ans (32\% avaient entre 22 et 29 ans, et $29 \%$ entre 30 et 39 ans) et avait entre un et dix ans d'expérience de travail dans leur fonction (52\%). Soixante sept pour cent (67\%) étaient des agents de comptoir, $17 \%$ des pharmaciens assistants et $16 \%$ des pharmaciens. Sur les 12 pharmaciens assistants enquêtés, $83 \%$ avaient obtenu leur doctorat en pharmacie. La majorité $(70 \%)$ des agents de comptoir interrogés $(\mathrm{n}=46)$ avaient terminé leurs études secondaires et 30\% avaient un diplôme supérieur. Selon la loi, un pharmacien titulaire doit avoir obtenu son Doctorat en pharmacie au Senegal. La plupart (59\%) des pharmacies privées avaient entre 3 et 5 employés, tandis que $41 \%$ avaient entre 6 et 8 employés (données non présentées).

Le Tableau 2 présente les réponses des participants concernant leur expérience avec l'offre des services de PF à l'enquête pré-test. Presque 35\% des pharmaciens et pharmaciens assistants avaient déjà participé à une formation en $\mathrm{PF}$, alors qu'un quart $(24 \%)$ des agents de comptoir avaient participé à une telle formation. Certains du personnel avait participé à une formation sur le counseling en PF avec 26\% des pharmaciens et pharmaciens assistants et $17 \%$ des agents de comptoir qui en ont bénéficiés. En ce qui concerne la pratique du counseling la PF aux clientes, $40 \%$ des pharmaciens et assistantes et $17 \%$ des agents de comptoir ont répondu affirmativement.

\section{TABLEAU 2 : EXPÉRIENCE D'OFFRE DES SERVICES DE LA PF AU PRÉ-TEST, $\mathrm{N}=69$}

\begin{tabular}{|c|c|c|}
\hline & $\%$ & $\mathbf{N}$ \\
\hline \multicolumn{3}{|l|}{ A déjà bénéficié d'une formation en PF } \\
\hline Pharmacien et pharmacien assistant $(n=23)$ & 34,8 & 8 \\
\hline Agent de comptoir $(n=46)$ & 23,8 & 11 \\
\hline \multicolumn{3}{|c|}{ A déjà bénéficié d'une formation sur le counseling PF } \\
\hline Pharmacien et pharmacien assistant $(n=23)$ & 26,1 & 6 \\
\hline Agent de comptoir $(n=46)$ & 15,2 & 7 \\
\hline \multicolumn{3}{|c|}{ A déjà fourni un counseling sur les méthodes de la PF } \\
\hline Pharmacien et pharmacien assistant $(n=23)$ & 39,1 & 9 \\
\hline Agent de comptoir $(n=46)$ & 17,4 & 8 \\
\hline
\end{tabular}




\section{RÉSULTAT 1 : CONNAISSANCES ET COMPÉTENCES DU PERSONNEL DE PHARMACIES}

\section{Connaissances du personnel des pharmacies sur le counseling en PF}

Le personnel des pharmacies a répondu à une série de questions sur leurs connaissances relatives au counseling en PF lors des enquêtes pré-test et post-test, à savoir : la connaissance des méthodes de PF ; ce qu'il faut discuter avec les clientes lorsqu'elles choisissent une méthode ; les questions pour exclure une grossesse ; l'espacement sain des naissances ; les méthodes de prévention du virus de l'immunodéficience humaine (VIH) ; et combien de jours après un rapport sexuel non protégé une femme peut utiliser des contraceptifs d'urgence.

L'évolution des connaissances du personnel des pharmacies sur les méthodes contraceptives entre l'enquête pré-test et l'enquête post-test est illustrée à la Figure 1. En général, le personnel des pharmacies connaissait très bien les méthodes contraceptives lors de l'enquête pré-test. Au moins $75 \%$ des participants ont pu identifier 10 des 12 méthodes contraceptives. Cinquante-neuf pourcent des participants ont identifié la vasectomie soit spontanément, soit à la suite d'une explication/ définition. À l'enquête du post-test, au moins $88 \%$ des participants ont identifié les 12 méthodes contraceptives. Cent pourcent des participants ont identifiés les préservatifs masculins et féminins, la pilule, les injectables, le DIU, la Méthode de l'allaitement maternel et de l'aménorrhée (MAMA) et le spermicide comme méthodes contraceptives dans l'enquête post-test.

Le Tableau 3 montre les connaissances du personnel des pharmacies sur les questions à poser au cours d'une séance de counseling en PF, à l'enquête prétest et à l'enquête post-test. Lors de l'enquête prétest, très peu de répondants $(9 \%)$ savaient qu'il faut demander aux clientes si elles voulaient des enfants à l'avenir, si elles ne souhaitaient pas utiliser certaines méthodes ou qu'elles n'ont pas bien tolérées dans le passé $(7 \%)$. Seul un personnel des pharmacies sur cinq s'était renseigné si la cliente avait des problèmes de santé ou si son partenaire la soutenait dans la PF.

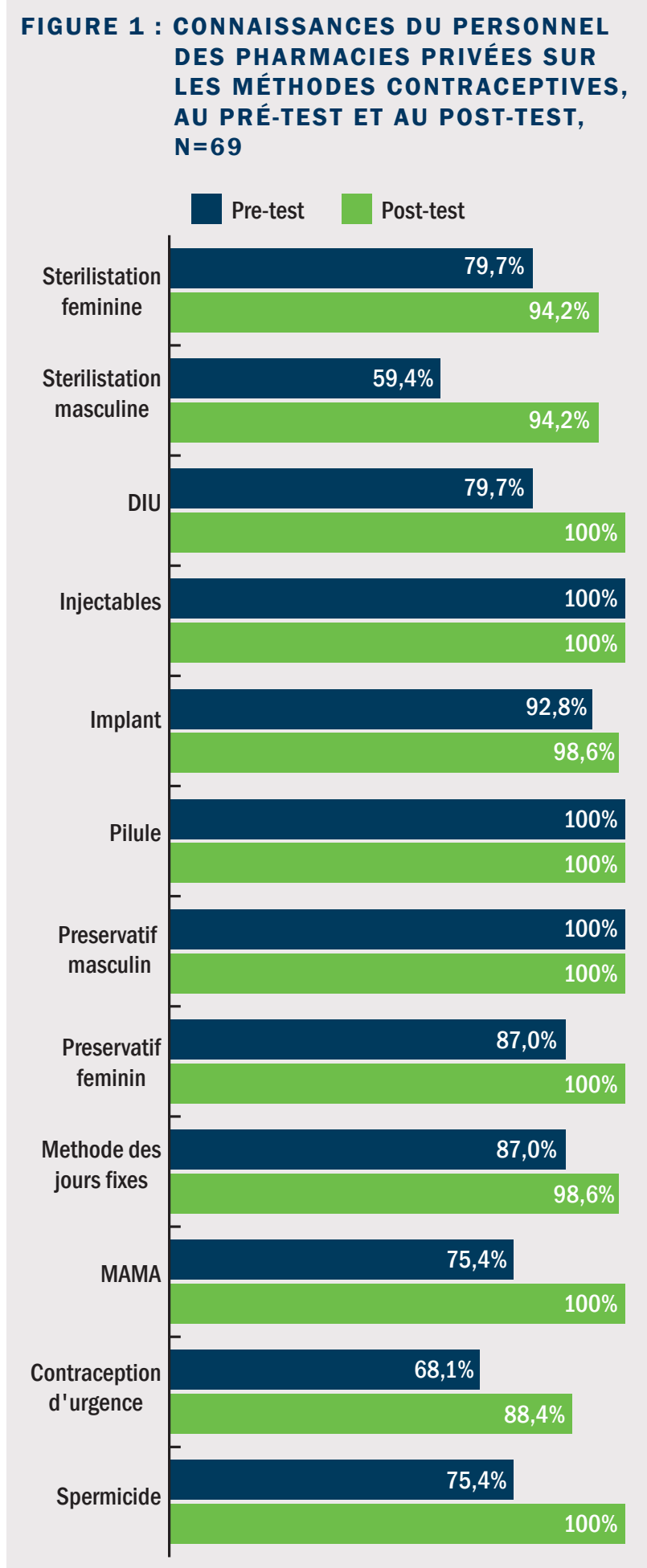


Les connaissances étaient encore faibles sur certains indicateurs de counseling en PF lors de l'enquête post-test. Moins de la moitié des participants ont pu identifier au moins une des questions clés. Par exemple, au post-test, seuls $46 \%$ des participants savaient qu'il faut demander si la cliente avait des problèmes de santé et seulement $16 \%$ savaient qu'il faut demander si la cliente voulait des enfants à l'avenir. De plus, la connaissance qu'il faut demander si le partenaire de la cliente la soutenait dans la $\mathrm{PF}$ à diminué passant de $22 \%$ au prétest à $16 \%$ au post-test.
TABLEAU 3 : CONNAISSANCES DU PERSONNEL DES PHARMACIES PRIVÉES SUR LES QUESTIONS CLÉS PENDANT UNE SÉANCE DE COUNSELING, AU PRÉ-TEST ET AU POST-TEST, $N=69$

\begin{tabular}{|c|c|c|}
\hline & $\begin{array}{c}\text { PRÉ-TEST } \\
(\%)\end{array}$ & $\begin{array}{c}\text { POST-TEST } \\
(\%)\end{array}$ \\
\hline Souhaitez-vous avoir des enfants à l'avenir? & 8,7 & 15,9 \\
\hline $\begin{array}{l}\text { Avez-vous accouché au cours des } 48 \\
\text { dernières heures? }\end{array}$ & 1,5 & 15,9 \\
\hline $\begin{array}{l}\text { Allaitez-vous un nourrisson de moins de } 6 \\
\text { mois? }\end{array}$ & 5,8 & 38,1 \\
\hline Votre partenaire vous soutient-il dans la PF? & 21,7 & 15,9 \\
\hline Avez-vous des problèmes de santé ? & 20,3 & 46,4 \\
\hline $\begin{array}{l}\text { Existe-t-il des méthodes que vous ne voulez } \\
\text { pas utiliser ou que vous n'avez pas bien } \\
\text { tolérées par le passé ? }\end{array}$ & 7,4 & 17,4 \\
\hline
\end{tabular}

\section{TABLEAU 4 : CONNAISSANCES DU PERSONNEL DES PHARMACIES PRIVÉES SUR LES QUESTIONS POUR ÉCARTER LE DOUTE D'UNE GROSSESSE, AU PRÉ-TEST ET AU POST-TEST, N=69}

\begin{tabular}{|c|c|c|}
\hline & $\begin{array}{c}\text { PRÉ-TEST } \\
(\%)\end{array}$ & $\begin{array}{c}\text { POST-TEST } \\
(\%)\end{array}$ \\
\hline Avez-vous eu un bébé de moins de 6 mois? & 8,7 & 37,7 \\
\hline $\begin{array}{l}\text { Vous êtes-vous abstenu de rapports } \\
\text { sexuels non protégés depuis vos dernières } \\
\text { menstrues ou votre accouchement? }\end{array}$ & 20,3 & 39,1 \\
\hline $\begin{array}{l}\text { Avez-vous accouché au cours des } 4 \\
\text { dernières semaines? }\end{array}$ & 4,4 & 17,4 \\
\hline $\begin{array}{l}\text { Vos menstrues ont-elles commencé au cours } \\
\text { des } 7 \text { derniers jours? }\end{array}$ & 59,4 & 66,7 \\
\hline $\begin{array}{l}\text { Avez-vous eu une fausse couche ou un } \\
\text { avortement ces } 7 \text { derniers jours? }\end{array}$ & 0,0 & 8,7 \\
\hline $\begin{array}{l}\text { Avez-vous correctement et } \\
\text { systématiquement utilisé une méthode } \\
\text { contraceptive fiable? }\end{array}$ & 1,5 & 8,7 \\
\hline
\end{tabular}

Le Tableau 4 décrit la connaissance sur des questions visant à exclure la grossesse. Au pré-test, les connaissances étaient faibles pour cinq des six questions elles sont restées faibles dans l'enquête post-test. Une connaissance élevée a été observée sur une seule question, notamment « vos menstrues ont-elles commencé au cours des 7 derniers jours ? » où $59 \%$ du personnel de la pharmacie lors du pré-test et $67 \%$ lors du post-test ont pu identifier cette question comme une question à poser aux clientes afin d'exclure une grossesse.

La Figure 2 montre les connaissances du personnel des pharmacies en matière de PF générale pour le counseling. Les

connaissances étaient élevées pour deux de ces indicateurs sur trois. Par exemple, lors de l'enquête pré-test, $73 \%$ du personnel a correctement identifié 24 mois comme étant la période recommandée par l'OMS pour un espacement des naissances sains et $96 \%$ savaient que les préservatifs sont une méthode contraceptive qui protège également contre les infections sexuellement transmissibles (IST) et le VIH. Cependant, aucun des participants ne savait qu'une femme pouvait prendre une contraception d'urgence jusqu'à cinq (5) jours après un rapport sexuel non protégé.

Selon l'enquête post-test, 94\% du personnel a identifié 24 mois comme étant la période recommandée pour un espacement sain des naissances et 100\% savaient que les préservatifs sont une méthode contraceptive qui protège contre les IST et le VIH. De même, 39\%, savaient qu'une femme peut utiliser un contraceptif d'urgence jusqu'à cinq jours après un rapport sexuel non protégé, comparé à $0 \%$ à l'enquête pré-test. Malgré cette hausse, les connaissances du personnel des pharmacies sur cet indicateur sont demeurées faibles. 


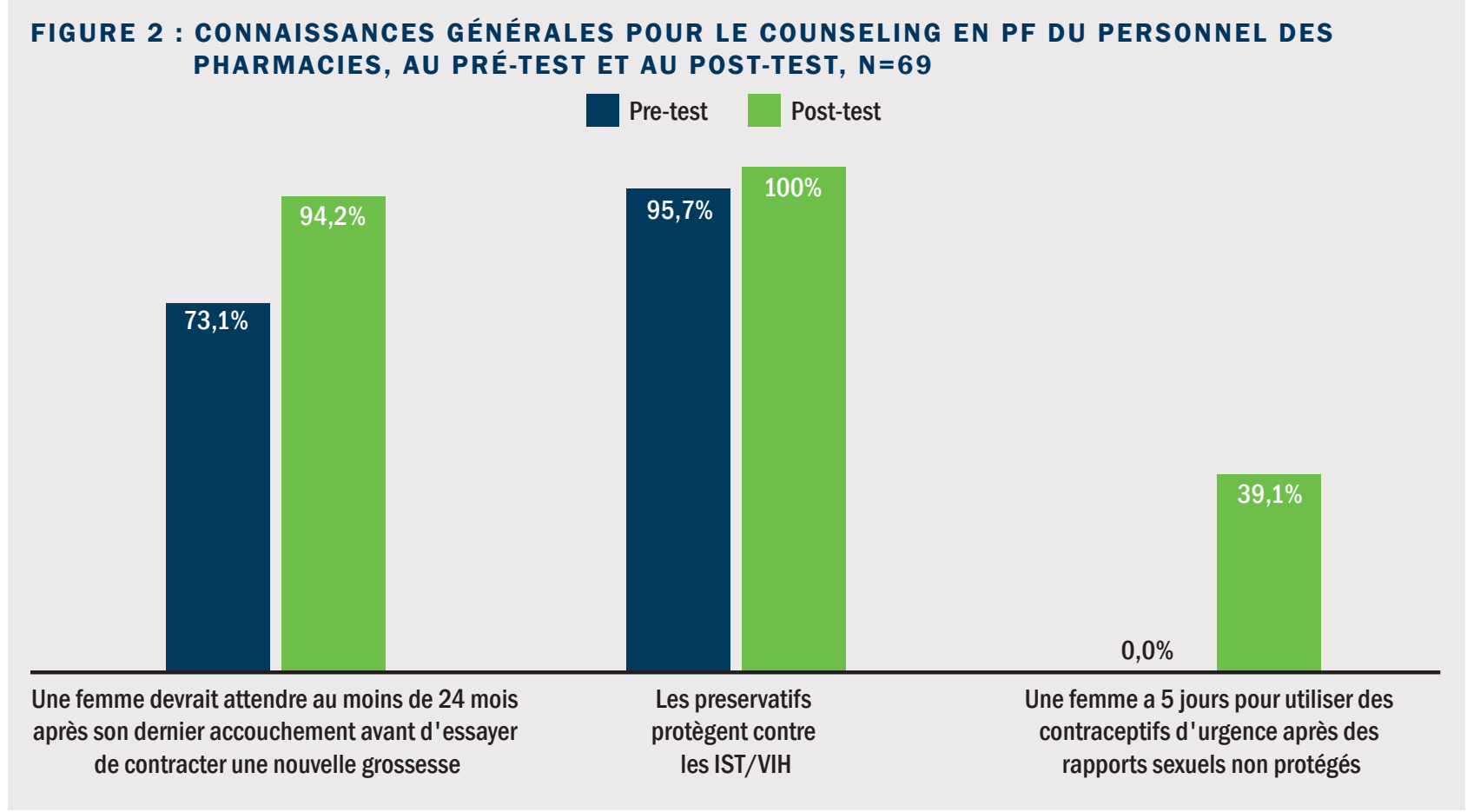

Connaissances des Pharmaciens et des Pharmaciens Assistants sur les Contraceptifs Oraux

Etant donné que les agents de comptoir n’ont pas participé aux séances sur les contraceptifs oraux, les résultats suivants concernent les pharmaciens et les assistants pharmaciens. Le Tableau 5 montreles connaissances des pharmaciens et des pharmaciens assistants sur les huit (8) effets secondaires courants des contraceptifs oraux combinés lors des enquêtes pré-test et post-test. À l'enquête pré-test, moins de $30 \%$ des participants ont été en mesure d'identifier 7 des 8 effets secondaires. Ce n'est que pour le « changement de poids » que $65 \%$ des répondants ont correctement identifié cet

TABLEAU 5 : CONNAISSANCE DES PHARMACIENS ET DES PHARMACIENS ASSISTANTS SUR LES EFFETS SECONDAIRES COURANTS DU CONTRACEPTIF ORAL COMBINÉ, AU PRÉ-TEST ET AU POST-TEST, $n=23$

\begin{tabular}{|lcc|} 
& $\begin{array}{c}\text { PRÉ-TEST } \\
(\%)\end{array}$ & $\begin{array}{c}\text { POST-TEST } \\
(\%)\end{array}$ \\
\hline Maux de tête & 30,4 & 82,6 \\
\hline Vertiges & 17,4 & 60,9 \\
\hline Nausée & 30,4 & 65,2 \\
\hline Lourdeur des seins & 0,0 & 17,4 \\
\hline Changements d'humeur & 0,0 & 4,4 \\
\hline Changement de poids & 65,2 & 78,3 \\
\hline Aménorrhée & 13,0 & 47,8 \\
\hline Saignements irréguliers & 43,5 & 30,4
\end{tabular}

effet secondaire lors de l'enquête pré-test.

A l'enquête post-test, des hausses ont été observées pour sept (7) des huit (8) effets secondaires. Au moins $61 \%$ des participants peuvent identifier les effets secondaires suivants : « maux de tête », " vertiges », « nausées » et « changement de poids». Les connaissances sont restées faibles (moins de la moitié) pour les quatre (4) autres effets secondaires : "lourdeur des seins», " changements d'humeur » « saignements irréguliers » et «aménorrhée ». 
Le Tableau 6 montre les connaissances des pharmaciens et des pharmaciens assistants sur les effets secondaires des contraceptifs oraux à progestatif seul à l'enquête pré-test et à l'enquête post-test. La connaissance des effets secondaires des contraceptifs oraux à progestatif seul était inférieure à celle des effets secondaires des contraceptifs oraux combinés. Lors des enquêtes prétest et post-test, moins de la moitié des pharmaciens et pharmaciens assistants ont pu identifier correctement cinq (5) des sept (7) effets secondaires. A l'enquête post-test $74 \%$ des participants ont identifié « maux de tête » et $52 \%$ ont identifié « nausées ».

Le Tableau 7 montre les connaissances des pharmaciens et des pharmaciens assistants en ce qui concerne les signes avant-coureurs qui exigent qu'une cliente se fasse soigner dans un établissement de santé. La connaissance de ces complications était très faible aux deux enquêtes. Un seul des cinq (5) signes avant-coureurs, « les maux de tête sévères ", a été connu par plus de $52 \%$ des participants au post-test. Aucun des participants n'a pu identifier de "problèmes de vision » ou de « douleurs aiguës aux jambes » comme complications, ni à l'enquête pré-test, ni à l'enquête post-test. Ces résultats suggèrent qu'une attention supplémentaire est nécessaire pendant la formation sur les signes avant-coureurs d'un contraceptif oral.

\section{TABLEAU 7 : CONNAISSANCE DES PHARMACIENS ET DES PHARMACIENS ASSISTANTS SUR LES SIGNES AVANT-COUREURS D'UN CONTRACEPTIF ORAL QUI EXIGENT QU'UNE CLIENTE SE FASSE SOIGNER DANS UN ÉTABLISSEMENT DE SANTÉ, AU PRÉ-TEST ET AU POST-TEST, $n=23$}

\begin{tabular}{|c|c|c|}
\hline & $\begin{array}{c}\text { PRÉ-TEST } \\
(\%)\end{array}$ & $\begin{array}{c}\text { POST-TEST } \\
(\%)\end{array}$ \\
\hline Douleur abdominale (forte) & 21,7 & 13,0 \\
\hline Douleur thoracique (sévère) & 0,0 & 13,0 \\
\hline Maux de tête (sévères) & 34,8 & 52,2 \\
\hline Problèmes oculaires (vision floue, brève perte de vision) & 0,0 & 0,0 \\
\hline Douleur aiguë dans la jambe & 0,0 & 0,0 \\
\hline
\end{tabular}

Le Tableau 8 montre les connaissances des pharmaciens et des pharmaciens assistants en ce qui concerne les 12 conditions qui limitent l'utilisation des contraceptifs oraux combinés. La connaissance de ces conditions était faible au pré-test- seulement $9 \%$ ou moins des 23 participants ont pu identifier l'une ou l'autre de ces 12 conditions. Les connaissances sur ces conditions sont demeurées faibles dans le cadre de l'enquête post-test, les connaissances élevées n'ayant été observées que pour deux conditions, 74\% des participants ont identifié «a des antécédents de cancer du sein » et 83\% ont identifié «la pression artérielle élevée ». Entre 35\% - 39\% des participants ont pu identifier « allaite un bébé de moins de 6 mois », « on leur a déjà dit qu'elle avait le diabète », « a deux ou plusieurs conditions susceptibles d'aggraver les risques de crise cardiaque ou d'AVC (tabagisme, obésité, diabète), et « on lui a déjà dit qu'elle avait une maladie rhumatismale comme le lupus ». Dix-sept pour cent ou moins des participants ont pu identifier les autres conditions. 
TABLEAU 8 : CONNAISSANCE DES PHARMACIENS ET DES PHARMACIENS ASSISTANTS SUR LES CONDITIONS QUI LIMITENT L'UTILISATION DES CONTRACEPTIFS ORAUX, AU PRÉ-TEST ET AU POST-TEST, $n=23$

\begin{tabular}{|c|c|c|}
\hline & $\begin{array}{l}\text { PRÉ-TEST } \\
(\%)\end{array}$ & $\begin{array}{l}\text { POST-TEST } \\
(\%)\end{array}$ \\
\hline Allaite un bébé de moins de 6 mois & 0,0 & 34,8 \\
\hline Fume des cigarettes et a plus de 35 ans & 8,7 & 8,7 \\
\hline A des antécédents de cancer du sein & 8.7 & 73,9 \\
\hline $\begin{array}{l}\text { A déjà eu un accident vasculaire cérébral, un caillot de sang dans la jambe ou les } \\
\text { poumons, ou une attaque cardiaque }\end{array}$ & 4,4 & 21,7 \\
\hline A des maux de tête sévères répétés & 4,4 & 17,4 \\
\hline Prend régulièrement des comprimés contre la tuberculose (TB) ou des crises? & 4,4 & 17,4 \\
\hline $\begin{array}{l}\text { A accouché au cours des } 6 \text { dernières semaines et présente les facteurs de risques } \\
\text { comme la prééclampsie, obésité et/ou tabagisme }\end{array}$ & 8,7 & 8,7 \\
\hline $\begin{array}{l}\text { A une maladie de la vésicule biliaire ou une maladie hépatique grave ou encore } \\
\text { l'ictère }\end{array}$ & 8,7 & 8,7 \\
\hline On leur a déjà dit qu'elle avait une pression artérielle élevée & 26,1 & 82,6 \\
\hline On leur a déjà dit qu'elle avait le diabète & 34,8 & 39,1 \\
\hline $\begin{array}{l}\text { A deux ou plusieurs conditions susceptibles d'aggraver les risques de crise cardiaque } \\
\text { ou d'AVC (tabagisme, obésité, diabète) }\end{array}$ & 17,4 & 39,1 \\
\hline On lui a déjà dit qu'elle avait une maladie rhumatismale comme le lupus & 4,4 & 39,1 \\
\hline
\end{tabular}

Le Tableau 9 montre les résultats de l'enquête pré-test et post-test sur les instructions à donner à une femme si elle oublie de prendre une (1) ou deux (2) pilules actives. Les connaissances des pharmaciens et des pharmaciens assistants étaient généralement plus élevées pour ces indicateurs. Dans le Tableau 9, il y a eu une augmentation des connaissances entre le pré-test et le post-test pour deux (2) des trois (3) instructions : 96\% des participants au post-test comparé à $39 \%$ des participants au pré-test ont identifié «prendre une pilule dès que possible » et $87 \%$ des participants au post-test comparé à 57\% des participants au pré-test ont identifié « continuer à prendre une pilule chaque jour ». Bien que ces résultats soient encourageants, aucun des participants à l'enquête pré-test ou post-test ne savait qu'une méthode de secours n'était pas nécessaire en cas d'oublie d'une ou deux pilules.

\section{TABLEAU 9 : CONNAISSANCE DE PHARMACIENS ET DE PHARMACIENS ASSISTANTS SUR LES INSTRUCTIONS À DONNER À UNE FEMME SI ELLE OUBLIE DE PRENDRE UNE PILULE "ACTIVE " 1 OU 2 JOURS D’AFFILÉE, AU PRÉ-TEST ET AU POST-TEST, n=23}

\begin{tabular}{|c|c|c|}
\hline & $\begin{array}{c}\text { PRÉ-TEST } \\
(\%)\end{array}$ & $\begin{array}{c}\text { POST-TEST } \\
(\%)\end{array}$ \\
\hline Prendre une pilule dès que vous vous souvenez & 39,1 & 95,7 \\
\hline Continuer à prendre une pilule chaque jour & 56,5 & 87,0 \\
\hline Pas besoin de protection contraceptive supplémentaire & 0,0 & 0,0 \\
\hline
\end{tabular}

Des résultats similaires ont été observés au Tableau 10 pour ce qui est de la conduite à tenir si une femme oublie trois (3) pilules actives ou plus. Presque tous les participants ont identifié trois (3) instructions à l'enquêtes post-test : 78\% ont identifié « prendre une pilule dès que possible » et ; 83\% ont identifié « continuer à prendre une pilule chaque jour » et " utiliser un condom ou s'abstenir lors de relations sexuelles ». Connaissance de l'instruction « commencer une nouvelle plaquette immédiatement» a diminué de $13 \%$ à $0 \%$ des participants entre le pré-test et le post-test et suggère qu'une attention supplémentaire est nécessaire pendant la formation sur cette instruction. 
TABLEAU 10 : CONNAISSANCE DES PHARMACIENS ET DES PHARMACIENS ASSISTANTS SUR LES INSTRUCTIONS À UNE FEMME SI ELLE OUBLIE UNE PILULE "ACTIVE " 3 JOURS OU PLUS D'AFFILÉE, AU PRÉ-TEST ET AU POST-TEST, n=23

\begin{tabular}{|c|c|c|}
\hline & $\begin{array}{c}\text { PRÉ-TEST } \\
(\%)\end{array}$ & $\begin{array}{c}\text { POST-TEST } \\
(\%)\end{array}$ \\
\hline Prendre une pilule dès que possible & 17,4 & 78,3 \\
\hline Continuer à prendre une pilule chaque jour & 47,8 & 82,6 \\
\hline Utiliser un préservatif ou s'abstenir de relations sexuelles & 30,4 & 82,6 \\
\hline $\begin{array}{l}\text { Commencer une nouvelle plaquette immédiatement si les pilules ont été oubliées au } \\
\text { cours de la troisième semaine }\end{array}$ & 13,0 & 0,0 \\
\hline
\end{tabular}

Connaissances des pharmaciens et des pharmaciens assistants sur les contraceptifs injectables

De même, les agents de comptoir n’ont pas participé aux séances sur les contraceptifs injectables. Les résultats suivants concernent donc seulement les pharmaciens et les assistants pharmaciens. Les pharmaciens et les pharmaciens assistants ont également reçu une formation sur les contraceptifs injectables à progestatif seul. Le Tableau 11 montre les connaissances des pharmaciens et des pharmaciens assistants sur les sept (7) effets secondaires courants des contraceptifs injectables lors des enquêtes pré-test et post-test. La connaissance de ces effets secondaires était généralement faible

TABLEAU 11 : CONNAISSANCE DES PHARMACIENS ET DES PHARMACIENS ASSISTANTS SUR LES EFFETS SECONDAIRES COURANTS DES CONTRACEPTIFS INJECTABLES, AU PRÉ-TEST ET AU POST-TEST, $n=23$

\begin{tabular}{|lcc|}
\hline Trouble des règles & $\begin{array}{c}\text { PRÉ-TEST } \\
(\%)\end{array}$ & $\begin{array}{c}\text { POST-TEST } \\
(\%)\end{array}$ \\
\hline Maux de tête & 65,2 & 82,6 \\
\hline Prise de poids & 13,0 & 34,8 \\
\hline Vertiges & 43,5 & 56,5 \\
\hline Retour tardif de la fécondité & 8,7 & 26,1 \\
\hline Irritation temporaire légère / & 0,0 & 17,4 \\
modérée de la peau & 0,0 & 17,4 \\
\hline Diminution de la libido & & \\
\hline
\end{tabular}
lors de l'enquête pré-test et est restée presqu'au même niveau lors de l'enquête post-test. Par exemple, seulement 35\% des participants ont été en mesure d'identifier des "maux de tête ", $26 \%$ ont identifié des "vertiges », et $17 \%$ ou moins ont identifié un « retour retardé à la fertilité », « une irritation temporaire de la peau » et « une diminution de la libido » comme effets secondaires des contraceptifs injectables lors de l'enquête post-test. A l'enquête pré-test et post-test, les connaissances étaient élevées pour deux (2) effets secondaires, «trouble des règles » (65\% des participants l'ont identifié au pré-test et $83 \%$ au post-test) et « la prise de poids » (44\% des participants l'ont identifié au pré-test et $57 \%$ au post-test).

Le Tableau 12 montre les connaissances des pharmaciens et des pharmaciens assistants sur les neuf (9) conditions qui limitent l'utilisation des contraceptifs injectables. La connaissance de ces conditions était faible lors des enquêtes pré-test et post-test. Bien qu'il y ait eu des hausses entre les enquêtes pré-test et post-test, les connaissances étaient élevées pour seulement deux conditions à l'enquête post-test : 67\% des participants ont identifié « le cancer du sein », et « l'hypertension artérielle » comme les conditions qui limitent l'utilisation des contraceptifs injectables à l'enquête post-test. Trente-cinq pour cent ou moins des participants ont été en mesure d'identifier les sept (7) autres indicateurs lors de l'enquête post-test. 

TABLEAU 12 : CONNAISSANCE DES PHARMACIENS ET DES PHARMACIENS ASSISTANTS SUR LES CONDITIONS QUI LIMITENT L'UTILISATION DES CONTRACEPTIFS INJECTABLES, AU PRÉ-TEST ET AU POST-TEST, $n=23$

\begin{tabular}{|c|c|c|}
\hline & $\begin{array}{l}\text { PRÉ-TEST } \\
(\%)\end{array}$ & $\begin{array}{l}\text { POST-TEST } \\
(\%)\end{array}$ \\
\hline On lui a dit qu'elle avait un cancer du sein & 0,0 & 69,6 \\
\hline $\begin{array}{l}\text { On lui a déjà dit qu'elle avait eu un accident vasculaire cérébral ou a présentement } \\
\text { un caillot de sang dans la jambe ou les poumons }\end{array}$ & 0,0 & 4,4 \\
\hline A une maladie hépatique grave ou la jaunisse (peau ou yeux jaunes) & 0,0 & 8,7 \\
\hline On lui a dit qu'elle souffrait de diabète (taux élevé de sucre dans le sang) & 17,4 & 17,4 \\
\hline On lui a dit qu'elle a une pression artérielle élevée & 13,0 & 69,6 \\
\hline $\begin{array}{l}\text { A saigné entre les périodes menstruelles, une situation inhabituelle pour elle (ou } \\
\text { saignement après des rapports sexuels) }\end{array}$ & 4,4 & 4,4 \\
\hline On lui a déjà dit qu'elle souffrait d'une maladie rhumatismale telle que le lupus & 0,0 & 34,8 \\
\hline $\begin{array}{l}\text { A deux ou plusieurs conditions qui pourraient aggraver les crises cardiaques ou } \\
\text { accidents vasculaires cérébraux (tabagisme, obésité, hypertension artérielle ou } \\
\text { diabète) }\end{array}$ & 30,3 & 34,8 \\
\hline Allaite actuellement un bébé de moins de quatre (4) semaines & 0,0 & 17,4 \\
\hline
\end{tabular}

Le Tableau 13 montre les connaissances des pharmaciens et des pharmaciens assistants concernant le moment où une femme peut commencer une méthode injectable à progestatif seul. Lors de l'enquête pré-test et posttest, les connaissances étaient très faibles avec seulement $30 \%$ ou moins des participants qui ont pu identifier l'un des 5 moments où une femme peut commencer une méthode injectable. Lors de l'enquête post-test $83 \%$ des répondants peuvent identifier qu'une femme peut commencer l'injectable « lorsqu'elle n'est pas enceinte ». Cela suggère qu'il faut mettre l'accent sur des interventions similaires dans le futur.

TABLEAU 13 : CONNAISSANCE DES PHARMACIENS ET DES PHARMACIENS ASSISTANTS SUR
LE MOMENT OÙ UNE FEMME PEUT COMMENCER À UTILISER DES MÉTHODES
INJECTABLES À PROGESTATIF-SEUL, AU PRÉ-TEST ET AU POST-TEST, $n=23$

\begin{tabular}{|c|c|c|}
\hline & $\begin{array}{c}\text { PRÉ-TEST } \\
(\%)\end{array}$ & $\begin{array}{c}\text { POST-TEST } \\
(\%)\end{array}$ \\
\hline Lorsqu'elle n'est pas enceinte & 26,1 & 82,6 \\
\hline Les 7 premiers jours d'un cycle menstruel sans méthode contraceptive & 30,4 & 30,4 \\
\hline $\begin{array}{l}\text { Après les } 7 \text { premiers jours d'un cycle menstruel, fournir une méthode contraceptive } \\
\text { pour les } 7 \text { premiers jours après l'injection }\end{array}$ & 4,4 & 4,4 \\
\hline Quatre semaines après l'accouchement & 17,4 & 30,4 \\
\hline Immédiatement après une fausse couche ou un avortement & 13,0 & 4,4 \\
\hline Immédiatement après l'arrêt d'une autre méthode & 26,1 & 4,4 \\
\hline
\end{tabular}

Les connaissances étaient généralement plus élevées pour les indicateurs spécifiques au DMPA-IM et au DMPA-SC. Le Tableau 14 illustre les connaissances des pharmaciens et des pharmaciens assistants sur le mode d'administration et le dispositif d'administration du DMPA-IM et du DMPA-SC. Trois (3) des quatre (4) indicateurs ont fait l'objet d'une amélioration des connaissances entre les deux enquêtes. Les participants qui ont correctement identifié le mode d'administration intramusculaire du DMPA-IM est passée de 52\% lors de l'enquête pré-test à $87 \%$ lors de l'enquête post-test. Les participants qui ont correctement identifié souscutanée comme mode d'administration du DMPA-SC est passée de 39\% lors de l'enquête pré-test à 100\% lors de l'enquête post-test. De même, les participants ayant identifié l'Uniject ${ }^{\mathrm{TM}}$ comme dispositif d'administration 
du DMPA-SC sont passés de 44\% lors de l'enquête pré-test à $65 \%$ au post-test. Bien qu'ils connaissent mieux le dispositif d'administration du DMPA-IM, qui est passé de $35 \%$ à $44 \%$, on constate que cette connaissance reste toutefois faible.

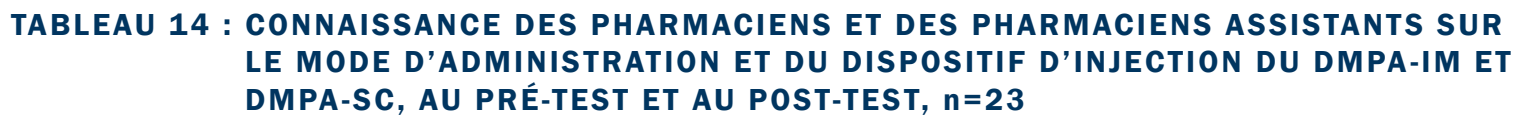

\begin{tabular}{|c|c|c|}
\hline & $\begin{array}{c}\text { PRÉ-TEST } \\
(\%)\end{array}$ & $\begin{array}{c}\text { POST-TEST } \\
(\%)\end{array}$ \\
\hline Mode d'administration de DMPA-IM (intramusculaire) & 52,2 & 87,0 \\
\hline Dispositif d'injection de DMPA-IM (seringue et aiguille) & 34,8 & 43,5 \\
\hline Mode d'administration du DMPA-SC (sous-cutanée) & 39,1 & 100,0 \\
\hline Dispositif d'injection du DMPA-SC (dispositif Uniject) & 43,5 & 65,2 \\
\hline
\end{tabular}

Dans le Tableau 15, des gains de connaissances ont également été observés entre l'enquête pré-test et celle du post-test concernant les connaissances des pharmaciens et des pharmaciens assistants des endroits sur le corps où le DMPA-IM et le DMPA-SC peuvent être injectés. Par exemple, les participants qui ont identifié le deltoïde comme un endroit pour administrer le DMPA-IM a augmenté à 100\% des personnes au posttest. Des résultats similaires ont été observés pour le DMPA-SC. Les participants qui ont identifié sous l'avant-bras est passée de $44 \%$ au pré-test à $91 \%$ à l'enquête post-test, les participants qui ont identifié l'abdomen est passée de 13\% à $91 \%$.

TABLEAU 15 : CONNAISSANCE DES PHARMACIENS ET DES PHARMACIENS ASSISTANTS SUR LES ENDROITS SUR LE CORPS OÙ LE DMPA-IM ET DMPA-SC SONT ADMINISTRÉS, AU PRÉ-TEST ET AU POST-TEST, $n=23$

\begin{tabular}{|lrr|} 
& \multicolumn{2}{c}{ PRE-TEST } \\
& $(\%)$ & $1 \%)$ \\
\hline Avant-bras (deltoide) DMPA-IM & 73,9 & 100,0 \\
\hline Fesses DMPA-IM & 30,4 & 65,2 \\
\hline Sous l'avant-bras DMPA-SC & 43,5 & 91,3 \\
\hline Dans l'adbdomen DMPA-SC & 13,0 & 91,3 \\
\hline Sur l'avant de la cuisse DMPA-SC & 13.0 & 78,3 \\
\hline
\end{tabular}

\section{Compétences en matière d'offre de méthodes}

L'évaluation des compétences des pharmaciens et des pharmaciens assistants sur l'offre des contraceptifs oraux et l'administration des injectables a été faite à partir d'observations de leur pratique en matière d'offre de contraceptifs oraux et de simulation sur des modèles pour l'administration des injectables (DMPA-IM et DMPA-SC). Cette évaluation a été réalisée avec un guide d'observation dans le cadre d'un suivi à un mois et demi après la formation qui leur a été dispensé par une formatrice. Au total, 23 pharmaciens et pharmaciens assistants ont pu être observés et ont eu trois (3) essais maximums pour démontrer leurs compétences. La compétence a été déterminé par un score de $80 \%$. Si un participant a reçu un score de moins de $80 \%$, la formatrice lui a donné des conseils pour prendre des mesures correctives et améliorer la technique. Pendant les visites du suivi, 23 des pharmaciens et pharmaciens assistants (100\%) ont démontré une compétence dans l'offre des contraceptifs oraux, 21 (91\%) ont démontré une compétence dans l'administration du DMPA-IM, et $23(100 \%)$ ont démontré une compétence dans l'administration du DMPA-SC (données non-présentées). 


\section{RÉSULTAT 2 : QUALITÉ DU COUNSELING EN PF À TRAVERS DES VISITES DE CLIENTES MYSTËRES}

L'offre de counseling en PF a été appréciée à travers l'approche des clientes mystères. La présente section fait le point sur la qualité de l'offre du counseling. Au total, les quatre (4) clientes mystères ont complété 52 visites au niveau des pharmacies. La cliente avec le profil de la pilule a visité 14 pharmacies, la cliente avec le profil de l'injectable a visité 13 pharmacies, la cliente avec le profil de la contraception d'urgence a visité 13 pharmacies et la cliente avec le profil de l'implant a visité 12 pharmacies. Le Tableau 16 montre la proportion du personnel des pharmacies avec qui les clientes mystères ont interagi. La moitié des clientes mystères a rencontré des pharmaciens assistants, $25 \%$ ont rencontré des pharmaciens et $25 \%$ a rencontré des agents de comptoir. Une cliente mystère a déclaré ne pas avoir reçu une séance du counseling en PF durant sa visite.

TABLEAU 17 : L'ENDROIT DU COUNSELING DE LA PF, SELON LES VISITES DES CLIENTES MYSTĖRES, $\mathrm{N}=51$

\begin{tabular}{|lc|}
\hline RUBRIQUE & $\%$ \\
\hline A reçu le counseling dans une salle isolée & 23,5 \\
\hline A reçu le counseling au niveau du comptoir & 58,8 \\
\hline A reçu le counseling dans un coin isolé derrière du comptoir & 17,7 \\
\hline Avait une intimité visuelle lors du counseling & 29,4 \\
\hline Avait une intimité auditive lors du counseling & 47,1 \\
\hline
\end{tabular}

\section{TABLEAU 16 : PROFIL DU PERSONNEL DES PHARMACIES RENCONTRÉ LORS DES VISITES DE CLIENTES MYSTÈRES, $\mathbf{N}=\mathbf{5 1}$}

\begin{tabular}{|c|c|}
\hline PROFIL & $\%$ \\
\hline Pharmacien & 25 \\
\hline Pharmacien assistant & 50 \\
\hline Agent de comptoir & 25 \\
\hline
\end{tabular}
conseillés dans une salle isolée comme l'exigent les procédures d'un counseling de qualité. Environ trois (3) clientes mystères sur cinq (5) ont reçu le counseling en PF au comptoir (58\%), ou dans un coin isolé derrière le comptoir $(17 \%)$. En effet, moins du tiers des clientes mystères $(29 \%)$ ont déclaré avoir eu une intimité visuelle et un peu moins de la moitié (47\%) ont déclaré avoir eu une intimité auditive.

Le Tableau 18 montre la proportion des clientes mystères qui ont déclaré que les aspects de counseling visant la qualité des services (Jain et al., 2019) leurs ont été posés sous forme de questions lors de leur session de counseling. La majorité des clientes ont déclaré que le personnel leur avait posé des questions sur leur expérience antérieure en $\mathrm{PF}(94 \%)$, leur préférence en matière de méthode de $\mathrm{PF}(82 \%)$ et leur ont parlé d'autres méthodes de PF (90\%). Dans 55\% des cas, les clientes mystères ont déclaré que le personnel leur a demandé si elles aimeraient avoir des enfants à l'avenir. Cependant, seulement 37\% des clientes mystères ont été interrogées sur le moment où elles aimeraient avoir des enfants. Environ un tiers des clientes mystères ont été informés des effets secondaires de leur méthode choisie (33\%) et de la possibilité de passer à une autre méthode si celle choisie était contre-indiquée (35\%). 
TABLEAU 18 : LES ASPECTS VISANT LA QUALITÉ DES SERVICES DANS LE PROCESSUS DU COUNSELING REÇU PAR LES CLIENTES MYSTĖRES LORS DE LEUR VISITES, N=51

RUBRIQUE

A demandé si la cliente aimerait avoir des enfants à l'avenir

A demandé quand la cliente aimerait avoir des enfants

A posé des questions sur l'expérience antérieure de la cliente en matière de PF

A posé des questions sur la préférence de la cliente en matière de la méthode de PF

A discuté des méthodes de PF avec la cliente

A informé la cliente sur les éventuels effets secondaires de la méthode choisie

A discuté avec la cliente des signes de danger liés à la méthode choisie

A dit ce que la cliente doit faire en cas de complications

A discuté avec la cliente la possibilité de passer à une autre méthode si celle choisie était 35,3 contre-indiquée

Le Tableau 19 montre les autres aspects généraux du counseling en PF. Dans la majorité des cas $(86 \%)$, les clientes mystères ont dit qu'on leur a posé des questions pour déterminer si oui ou non elles étaient enceintes. Dans environ deux tiers des cas, elles ont déclaré que le personnel de la pharmacie leur ont dit comment utiliser la méthode choisie (70\%). Par contre, moins de la moitié des clientes ont été interrogées sur la question de savoir si leur partenaire les soutenait dans la PF $(47 \%)$ ou si elles avaient des problèmes de santé ou prenaient des médicaments (49\%). Dans un peu moins d'un quart des cas (24\%), il leur a été demandé s'il existe des méthodes qu'elles ne voulaient pas utiliser ou n'avaient pas bien tolérées dans le passé.

Lorsqu'on leur a demandé si le personnel utilisait les aide-mémoires pendant la séance de counseling, les clientes mystères ont répondu oui dans $48 \%$ des cas (49\% pour le BCS+ et $47 \%$ pour le guide MEC de l'OMS, données non présentées).

\section{RÉSULTAT 3 : OFFRE DES SERVICES EN PF ET GESTION DES DONNÉES}

Un mois et demi après la formation, 68 personnels des pharmacies ont été enquêtés sur les services fournis depuis la formation (22 pharmaciens et pharmaciens assistant et 46 agents de comptoir). Le Tableau 20 montre les services que le personnel des pharmacies a déclaré avoir offert lors de l'enquête de suivi. La majorité (77\%) de pharmaciens et pharmaciens assistants ont déclaré avoir fait le counseling en PF, alors que 44\% des agents de comptoir ont déclaré la même chose. La plupart (68\%) des pharmaciens et pharmaciens assistants ont déclaré avoir conseillé des clientes sur l'utilisation de contraceptifs oraux, contre 44\% des agents de comptoir.

La majorité du personnel a estimé que leur pharmacie disposait d'un espace adéquat pour assurer aux clientes la confidentialité des conversations $(82 \%$ des pharmaciens et pharmaciens assistants et $76 \%$ des agents de comptoir) et la confidentialité visuelle ( $82 \%$ des pharmaciens et pharmaciens assistants et $73 \%$ des agents de 
comptoir). Malgré ces résultats, la plupart des clientes mystères n'avaient pas l'impression d'avoir une intimité visuelle ou auditive (voir les résultats ci-dessus). Tous les membres du personnel ont trouvé utiles les aidemémoires de counseling BCS+ et MEC fournis pendant la formation.

TABLEAU 20 : OFFRE DES SERVICES DE LA PF, ENQUÊTE DU SUIVI, $n=68$

\begin{tabular}{|c|c|c|}
\hline & $\%$ & $n$ \\
\hline \multicolumn{3}{|c|}{ A déjà fourni un counseling sur les méthodes de PF } \\
\hline Pharmacien/Pharmacien assistant & 77,3 & 17 \\
\hline Agent du comptoir & 43,5 & 20 \\
\hline \multicolumn{3}{|c|}{ A déjà offert un counseling à un client sur l'utilisation des contraceptifs oraux } \\
\hline Pharmacien/Pharmacien assistant & 68,2 & 15 \\
\hline Agent du comptoir & 43,5 & 20 \\
\hline \multicolumn{3}{|c|}{ La pharmacie dispose un espace adéquat y compris l'intimité auditive } \\
\hline Pharmacien/Pharmacien assistant & 81,8 & 18 \\
\hline Agent du comptoir & 76,1 & 35 \\
\hline \multicolumn{3}{|c|}{ La pharmacie dispose un espace adéquat y compris l'intimité visuelle } \\
\hline Pharmacien/Pharmacien assistant & 81,8 & 18 \\
\hline Agent du comptoir & 73,1 & 34 \\
\hline \multicolumn{3}{|c|}{ Les aide-mémoires de counseling que j'ai reçu lors de la formation sont utiles } \\
\hline Pharmacien/Pharmacien assistant & 100,0 & 22 \\
\hline Agent du comptoir & 100,0 & 46 \\
\hline
\end{tabular}

La Figure 3 illustre les perceptions de la durée du counseling en PF parmi le personnel qui, après la formation a fait du counseling. Selon les 37 personnels des pharmacies qui ont fait le counseling en PF, $60 \%$ ont déclaré qu'ils estimaient avoir passé une durée appropriée à conseiller les clientes. Trente-cinq pour cent (35\%) ont trouvé qu'ils ont passé trop de temps et $5 \%$ ont déclaré qu'ils n'ont pas passé assez de temps à conseiller les clientes. La majorité $(86 \%)$ de ces personnels a déclaré qu'elle peut faire le counseling en plus de ses autres activités quotidiennes. Parmi tout le personnel enquêté ( $\mathrm{N}=68), 78 \%$ étaient confiants de pouvoir conseiller les clientes sur l'utilisation des contraceptifs oraux (données non présentées).

FIGURE 3 : PERCEPTION DE LA DURÉE DU COUNSELING EN PF PARMI LE PERSONNEL QUI A FAIT LE COUNSELING EN PF APRÈS LA FORMATION, $n=37$

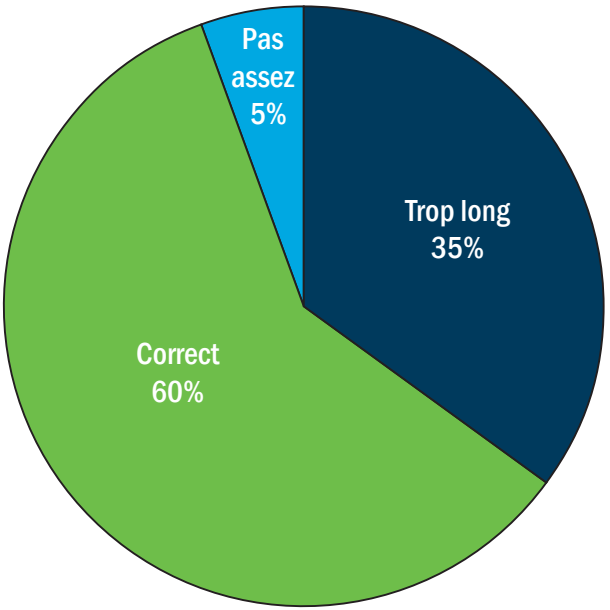

Comment appréciez-vous le temps que vous consacrez pour faire le counseling à une cliente?

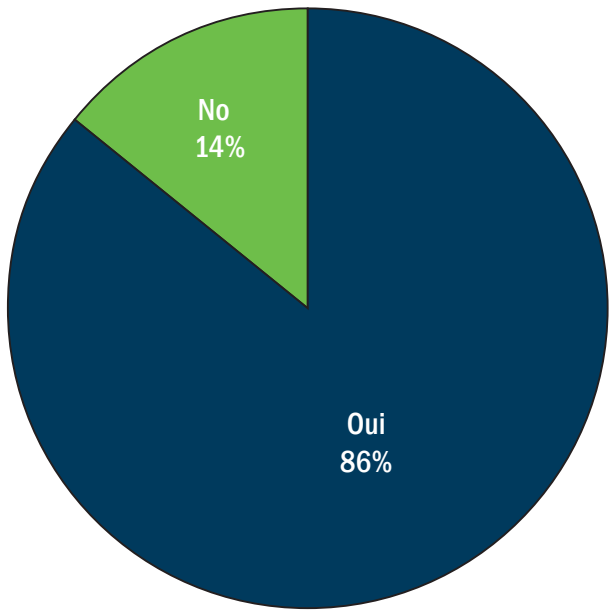

Parvenez-vous à faire le counseling en plus de vos autres activités quotidiennes? 
Environ 1 mois et demi après la formation, le personnel a reçu une visite de l'équipe de recherche et les formateurs dans leur pharmacie pour évaluer la qualité de la gestion des données et discuter avec le personnel de leurs impressions sur la prestation de conseils en PF aux clientes, en plus de leurs responsabilités quotidiennes. L'équipe de recherche et les formateurs ont visité 14 pharmacies privées. Sur ces 14, deux (2) pharmacies n'avaient pas de dossiers remplis. Sur les 12 autres, 7 pharmacies avaient des dossiers complets et 6 l'avaient fait avec précision. Par conséquent, environ la moitié des pharmacies avait conservé des dossiers complets et précis sur la PF au moment de la visite de suivi d'un mois et demi. Toutes les pharmacies ont été observées en train d'entreposer ces dossiers dans un endroit sécurisé. 


\section{Discussions}

\section{POTENTIEL DE COUNSELING EN PF DANS LES PHARMACIES PRIVÉES AVEC UN SOUTIEN SUPPLÉMENTAIRE ET CONTINU}

Les résultats de cette étude suggèrent que du travail supplémentaire et des approches innovantes sont nécessaires pour mieux outiller le personnel des pharmacies privées dans l'offre des services de PF. Le personnel des pharmacies avait généralement une faible connaissance en PF, ce qui est conforme aux résultats antérieurs (Mbow 2018; Fall, 2015). En ce qui concerne le counseling en PF, les connaissances du personnel (pharmaciens, pharmaciens assistant et agents de comptoir) sur les questions clés de counseling et les questions visant à exclure la grossesse étaient faibles avant et même après la formation sur la base des résultats des enquêtes. Cependant, le personnel des pharmacies connaissait presque toutes les méthodes disponibles au Sénégal et certaines informations clés pour les clientes, telles que les méthodes qui protègent également contre le VIH et les IST et le temps recommandé pour l'espacement des naissances.

Si l'on se base sur l'analyse de données des clientes mystères, le personnel des pharmacies a montré un certain potentiel. Par exemple, dans la plupart des cas, les clientes mystères ont été interrogées sur leur expérience en PF, ont été informées des autres méthodes de PF, ont été interrogées sur la méthode qu'elles préféreraient utiliser, et ont été orientées sur la façon d'utiliser la méthode de leur choix. Néanmoins, un appui supplémentaire de manière générale est nécessaire pour s'assurer que le personnel fourni de façon constante des services de counseling de qualité. Peu de clientes mystères ont été informées des effets secondaires, des signes de danger et des mesures à prendre si elles rencontrent des problèmes avec leur méthode. Des efforts restent à faire en ce qui concerne l'information sur les éventuels effets secondaires qui sont souvent causes de discontinuation, voire d'abandon et, sur la conduite à tenir en cas de survenue de complications.

Par ailleurs, la plupart des clientes mystères ont été conseillées au comptoir de la pharmacie et peu d'entre elles ont perçu une intimité auditive ou visuelle. Des efforts restent également à faire pour le respect des normes de confidentialité requises pour faire un bon counseling. Ainsi, ces conditions d'administration du counseling révèlent le manque de respect des règles de confidentialité qui sont fondamentales pour cette étape cruciale de l'offre de services de PF. La surcharge de travail du personnel a été avancée comme facteur justifiant les circonstances de telles pratiques.

En résumé, l'analyse de résultats de cette étude indiquent un potentiel de la pratique du counseling dans les pharmacies enrôlées. Toutefois, des efforts méritent d'être consentis pour respecter les normes de qualité requises pour des services de bonne qualité et un accompagnement effectif des clientes.

\section{LES PHARMACIENS ET LES PHARMACIENS ASSISTANTS ONT BESOIN D'UN SOUTIEN SUPPLÉMENTAIRE POUR MIEUX OFFRIR DES CONTRACEPTIFS ORAUX ET INJECTABLES}

Les pharmaciens et les pharmaciens assistants ont des connaissances faibles sur les principaux indicateurs des contraceptifs oraux et injectables. Par exemple, même après la formation, peu de pharmaciens et de pharmaciens assistants ont correctement identifié les effets secondaires courants, les conditions qui limitent l'utilisation des contraceptifs oraux, et les signes avant-coureurs des contraceptifs oraux. Des résultats similaires ont été observés pour des informations clés sur les contraceptifs injectables pour lesquels les connaissances sur les effets secondaires, les conditions qui limitent son utilisation et quand une femme peut commencer l'injection étaient faibles. En plus des connaissances faibles, 56\% des clientes mystères qui ont été reçues par un pharmacien ou un pharmacien assistant ont déclaré que leur prestataire a utilisé l'aide-mémoire BCS+ et 53\% 
ont déclaré que leur prestataire a utilisé l'aide-mémoire MEC. Donc, seulement la moitié des pharmaciens et des pharmaciens assistants utilisent des aide-mémoires pour s'assurer qu'ils fournissent des informations complètes sur les effets secondaires ou qu'ils effectuent un dépistage adéquat des clients.

Toutefois, les pharmaciens et les pharmaciens assistants ont montré qu'ils savaient bien comment expliquer aux femmes l'utilisation des contraceptifs oraux. Les connaissances étaient satisfaisantes pour les indicateurs spécifiques au DMPA-IM et au DMPA-SC, y compris le mode d'administration et les endroits où chaque méthode peut être administrée sur le corps.

Ces résultats suggèrent qu'un travail supplémentaire est nécessaire pour aider les pharmacies privées à mieux offrir le counseling et des contraceptifs oraux. En outre, les résultats suggèrent qu'il y a également une opportunité pour les pharmacies privées d'offrir les injectables, mais des interventions supplémentaires, parmi lesquelles la mise en œuvre de programmes ou recherches, sont nécessaires pour la formation et le soutien appropriés. Dans tous les cas, l'accent mis sur le dépistage des clientes pour s'assurer qu'elles sont éligibles sur le plan médical sera crucial pour les interventions futures. De plus, il est également nécessaire de mettre l'accent sur la fourniture aux clientes d'informations complètes sur les effets secondaires.

\section{DÉFIS}

La mise en œuvre de l'étude a permis de constater que pour permettre au personnel des pharmacies d'offrir des services de PF de qualité, il faudra relever certains défis :

\section{La formation}

L'équipe de recherche a été confrontée à des difficultés pour l'organisation des sessions de formation. Bien que toutes les dispositions aient été prises durant la phase préparatoire pour garantir la participation effective du personnel des pharmacies aux sessions (réduction de la durée initiale de la formation de 4 jours de 8 heures [32 heures au total] à 3 jours de 5 heures [15 heures au total], possibilité de choisir sur une période donnée, celle qui leur convenait le mieux) plusieurs cas de non disponibilité ont été notées au moment de la formation, notamment de la part des pharmaciens titulaires. Ceci ayant occasionné l'exclusion de certains participants qui avaient été enrôlés au début de l'intervention. De plus, la courte durée de la formation n'a pas pu offrir assez d'espace pour toutes les éventuelles questions et une durée de pratique plus longue, et à cause de cela d'ailleurs, certaines séances de formation ont dépassé le temps alloué. Par conséquent, des approches d’apprentissage innovantes qui respectent les horaires des pharmaciens doivent être explorées.

\section{La confidentialité de l'offre de services dans les pharmacies privées}

Bien que toutes les pharmacies visitées disposent d'un espace qui pourrait assurer la confidentialité de l'offre de services de PF, le personnel des pharmacies ne s'est pas encore approprié le comportement adéquat qui consiste à s'isoler avec la cliente pour une interaction efficace. Ainsi il convient de promouvoir l'importance de la confiance en soi et envisager des interventions visant des changements sociaux de comportement.

\section{Le suivi et la supervision des pharmacies privées}

Pour renforcer les connaissances et compétences du personnel des pharmacies privées et assurer la qualité des services, l’offre de services de PF par les pharmacies privées doit, comme pour toute délégation de tâches, être suivie et supervisée conformément aux politiques, normes et protocoles. Toutefois, cette supervision est tributaire de la disponibilité des formateurs et des ressources mises en place à cet effet. 


\section{LIMITES DE L'ÉTUDE}

La durée de l'intervention constitue la principale limite de cette étude. Compte tenu des contraintes de temps pour l'obtention de l'avis éthique, la durée de l'intervention a été réduite et par conséquent le nombre de visites de suivi aussi. Une période de 6 à 8 mois ou à défaut de 4 à 5 mois comme initialement prévu aurait permis de suivre sur une période raisonnable la qualité des services offerts et de fournir un appui continu au personnel des pharmacies.

Deuxièmement, la législation du Sénégal empêche les pharmacies privées de faire des injections. Par conséquent, les pharmacies privées qui ont participé à l'étude n'ont pas administré d'injectables à leurs clientes qui sont interessées par cette méthode. Bien que les pharmaciens et pharmaciens assistants soient formés sur le sujet et qu'ils aient pratiqué leurs compétences sur des mannequins, la motivation à participer pleinement à l'ensemble de la formation était faible peut-être parceque le personnel formé n'avait pas l'opportunité de mettre en pratique sur des clientes réelles ce qu'il avait appris.

Enfin, face aux obstacles dans la mise en œuvre de l'étude mentionnés dans ce rapport, la taille de l'échantillon a été de 69 personnels des pharmacies, dont seulement 23 pharmaciens et pharmaciens assistants. Une telle taille d'échantillon ne permet ni de faire des tests de signification statistiques (ex. pour les résultats des contraceptifs oraux et injectables) ni de généraliser les résultats.

\section{IMPLICATIONS ET RECOMMANDATIONS}

Le personnel des pharmacies constitue pour les populations une source potentielle de services de counseling, de contraceptifs oraux et de services d'injectables. Des interventions supplémentaires, menées dans un environnement juridique favorable, seraient nécessaires pour apporter des évidences supplémentaires sur l'offre de contraceptifs injectables par des pharmacies privées aux clientes réelles. Les résultats de l'étude montrent qu'ils peuvent acquérir des connaissances et des compétences, mais cela exige un soutien supplémentaire pour renforcer et maintenir ces connaissances dans la durée. Les résultats de cette étude viennent compléter ceux de la phase exploratoire menée en 2016 et fournissent au Ministère de la santé des évidences additionnelles de la faisabilité de l'offre de services de PF par les pharmacies privées, même si leurs compétences doivent être renforcées. Ces résultats peuvent aussi contribuer au dialogue national sur les politiques relatives de la délégation des tâches au secteur privé.

Sur la base de ces constats, les recommandations suivantes ont été formulées :

\section{Concernant l'environnement juridique}

- Explorer les voies et moyens pour la mise en place d'un environnement juridique favorable et la mise en oeuvre d'interventions qui s'avèrent nécessaires avec les pharmacies privées sur l'offre d'injectables aux clientes de PF volontaire.

- Mener d'autres interventions sur l'offre de services injectables dans un contexte juridique favorable.

\section{Concernant la mise à l'échelle}

- Etendre l'expérience aux autres pharmacies privées, notamment au niveau des autres régions en tenant compte des aspects à renforcer.

- Utiliser les résultats de cette étude pour faire avancer le dialogue national avec toutes les parties prenantes sur l'implication des pharmacies privées dans l'offre de services de PF. 


\section{Concernant la formation}

- Explorer, en collaboration avec l'OPS et le syndicat des pharmaciens, de nouvelles approches de formation telles que les formations sur sites (au sein même des pharmacies) ou des formations durant les week-ends.

- Explorer les délégués médicaux comme un moyen de renforcer la connaissance de la PF avec les pharmaciens privés.

- Standardiser la durée des formations à un minimum de 32 heures pour parcourir le curriculum au complet et pour avoir suffisamment de temps à consacrer à la pratique, notamment en ce qui concerne le respect des normes et procédures.

- Examiner quelles sont les aide-mémoires qui soutiennent le mieux l'offre de services de PF par les pharmacies privées, y compris si un outil standardisé pourrait soutenir les pharmacies privées.

\section{Concernant la confidentialité de l'offre de services dans les pharmacies privées}

- Encourager les pharmaciens à aménager un espace adéquat pour le counseling en PF.

- Inclure dans la formation un volet spécifique sur les politiques, normes et protocoles de l'offre de services de PF.

- Explorer les interventions du changement social de comportement pour aider le personnel des pharmacies privées à fournir des services dans un espace isolé et non au comptoir.

\section{Concernant le suivi/supervision des pharmacies privées}

- Mettre en place des stratégies permettant au personnel des pharmacies d'acquérir des compétences additionnelles telles que la formation continue et/ou les supervisions formatives.

- Impliquer l'OPS dans le développement d'un système de supervision et de soutien technique pour les pharmaciens et pharmaciens assistants dans l'offre de la PF. Institutionaliser un système de supervision de l'assurance qualité avec l'OPS et le syndicat des pharmaciens directement impliqués dans l'évaluation régulière de la qualité dans l'offre de la PF. 


\section{Références}

Agence Nationale de la Statistique et de la Démographie (ANSD) [Sénégal], et ICF. 2016. Sénégal : Enquête Démographique et de Santé Continue (EDS-Continue 2015). Rockville, Maryland, USA: ANSD et ICF International.

Agence pour la Promotion des Activités de Population - Sénégal (APAPS). 2016. Measurement, Learning and Evaluation Project (MLE), Initiative Sénégalaise de Santé Urbaine (ISSU).

Cadre stratégique national pour la planification familiale. 2016-2020. Ministère de la Santé et de l'Action sociale.

Diop Nafissatou J; Mane Babacar; Faye Ousseynou; Niang Cheikh Ibrahima; Thiaw Thiaba; Sano Coly Aissatou; Fatou Bintou Mbow; Niang Faye Youmane. 2009. Enquête sur la contraception d'urgence (CU) au niveau. des pharmacies.

Fall, C. and M. Diop. 2015. Enquête sur la planification familiale dans les pharmacies du milieu urbain au Sénégal 2015. Rapport d 'analyse FHI 360 (unpublished).

Fitzpatrick, A, and K. Tumlinson. 2017. «Strategies for optimal implementation of simulated clients for measuring quality of care in low- and middle-income countries. » Glob Health Sci Pract. 5(1): 108-114. doi: 10.9745/GHSP-D-16-00266

Initiative Sénégalaise de Santé Urbaine. 2014. Planification Familiale Initiative Sénégalise de Santé Urbaine «ISSU » Capitalisation des interventions du projet ISSU. www.issu.sn

Jain, A. et al. 2019. «Validation of two quality of care measures: results from a longitudinal study of reversible contraceptive users in India, » Studies in Family Planning 50(2): 179-193. doi: 10.1111/sifp.12093

Loi n 54-418 du 15 avril 1954 étendant aux territoires d'Outre-Mer, au Togo et au Cameroun certaines dispositions du Code de la Santé Publique relatives à l'exercice de la pharmacie.

Pratiques à haut impact dans la planification familiale (PIH). 2013. Dépôts pharmaceutiques et pharmacies : des sources d'information et d'approvisionnement en produits de planification familiale. Washington, DC: USAID. Disponible à l'adresse suivante. http://www.fphighimpactpractices.org/resources/drug-shops-andpharmacies-sources-family-planning-commodities-and-information.

Mbow, Fatou Bintou, Sara Chace Dwyer, et Nafissatou Diop. 2018. "Exploration du rôle potentiel des pharmacies privées dans l'offre de services de planification familiale au Sénégal : Synthèse de l'étude," Synthèse. Dakar, Sénégal: Population Council, Le Projet Evidence.

PATH. 2014. Sayana ${ }^{\circledR}$ Press Clinical Brief. Frequently asked questions about Sayana® Press. www.path.org

Plan d'Action National de Planification Familiale 2012-2015. République du Sénégal. Dakar, Sénégal: MSAS.

Population Council. 2015. The Balanced Counseling Strategy. A Toolkit for Family Planning Service Providers Working in High STI/HIV Prevalence Settings. 3rd edition. https://www.popcouncil.org/research/thebalanced-counseling-strategy-plus-a-toolkit-for-family-planning-service 


\section{Annexe 1}

\section{L'AFFICHE DE LA DEMANDE}

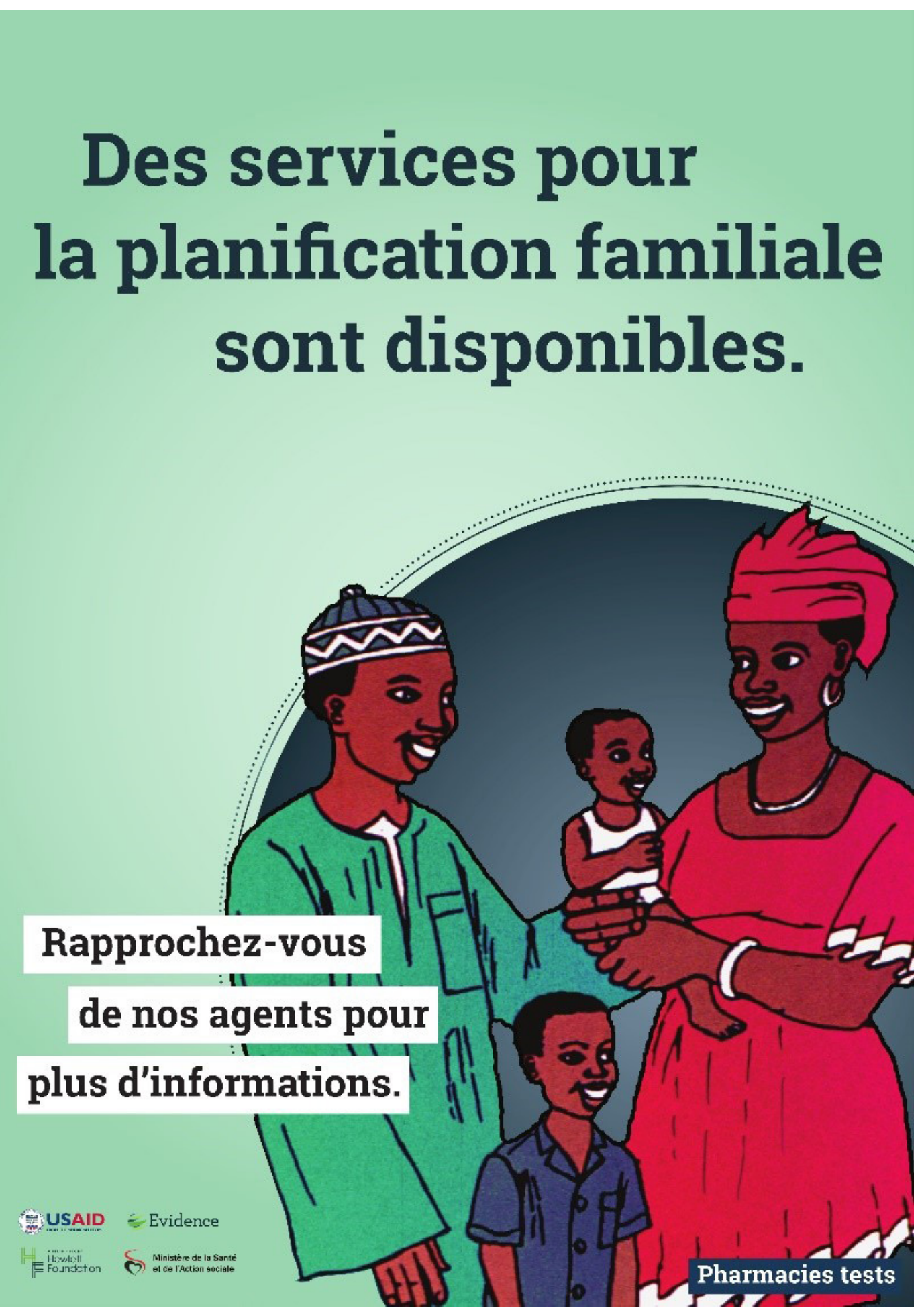




\section{The Evidence Project}

Population Council

4301 Connecticut Avenue, NW, Suite 280

Washington, DC 20008 USA

tel +12022379400

evidenceproject.popcouncil.org 